\title{
A Novel Representative $k$-NN Sampling-based Clustering Approach for an Effective Dimensionality Reduction-based Visualization of Dynamic Data
}

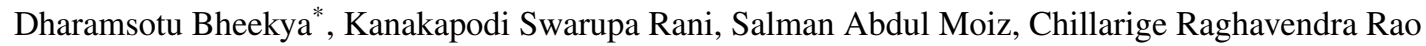

School of Computer and Information Sciences, University of Hyderabad, Hyderabad, Telangana, 500046, India.

\begin{tabular}{l} 
A R T I C L E I N F O \\
\hline Article history: \\
Received: 13 May, 2020 \\
Accepted: 19 June, 2020 \\
Online: O6 July, 2020 \\
\hline Keywords: \\
Dimensionality Reduction \\
Exploratory Analysis \\
Visualization \\
Clustering \\
Sampling \\
-Distributed Stochastic Neigh- \\
bor Embedding \\
Cluster Validation Index \\
Interpolation
\end{tabular}

\section{Introduction}

Exploratory analysis of Big Data is ubiquitous in an increasing number of fields and vital to their progress. Visualization plays a paramount role in an exploratory study. Data visualization is applicable for the limited number of dimensions, which depends on the perceptual capability of the analyst. For exploratory analysis, traditional visualization techniques may not provide useful visual insights of high-dimensional data and they are restricted for a limited number of dimensions. The conventional feature selection methods may not provide helpful visual insights for exploratory analysis, which happens due to the inappropriate feature selection. There is a requirement of the feature extraction technique, which shows the correlation between the original features of the data. The Dimensionality Reduction (DR) technique transforms the data and extract new features, which makes data analysis tractable. From the last few decades, researchers have proposed various linear as well as non-linear DR techniques in the context of visualization. The linear DR techniques like Principal Component Analysis (PCA) [1], Multidimensional Scaling (MDS) [2] and Factor Analysis (FA) [3] deals with the simple structured data. It is challenging to extract valuable information from complex structured data using linear DR. In contradiction to linear DR, the non-linear DR techniques like Isomap [4], Local Linear Embedding (LLE) [5], Laplacian Eigenmap (LE) [6] and Stochastic Neighbor Embedding (SNE) [7] deals with non-linear data. The paper [8] provides a complete comparative study of DR techniques. The methodology of the DR technique depends on its feature extraction criteria. The feature extraction depends on the characteristics of interest in the data such as interpoint distances, reconstruction weights, variation, linear subspace, geodesic distances, linear tangent space, neighborhood graph and conditional probability distribution.

Among all the DR techniques, t-Distributed Stochastic Neighbor Embedding (t-SNE) [9], is an improved version of SNE, introduced by Laurens Van Der Maaten and Geoffrey Hinton in 2008. The $\mathrm{t}-\mathrm{SNE}$ has gained impressive attention and enormous popularity in several fields [10,12]. The empirical study states that the lowdimensional visual representation of t-SNE is more robust than any other DR technique. The t-SNE algorithm most commonly used to preserve the original structure of the high-dimensional data in

*Corresponding Author: Dharamsotu Bheekya, +919573466965, \& 15mcpc21@uohyd.ac.in 
very low-dimensional embedding (i.e., either 2D or 3D). From past several years the $\mathrm{t}-\mathrm{SNE}$ has been explored in various aspects such as optimization [13]|14], scalability [14-17], dealing with non-numeric data [18], outliers separation [17] and many more.

The t-SNE is a non-parametric technique that provides flexibility in learning and reduces computational complexity. The nonparametric nature limits t-SNE applicability to the out-off-sample extension, which means the addition of new data samples into the existing $\mathrm{t}$-SNE environment is not possible. If we want to add a new sample, then we should re-run the entire t-SNE model by including a new sample. When the addition of new data points increases, the computational cost of t-SNE also increases monotonically. Therefore, it does not apply to time-series and streaming data The LION-tSNE [17] of Boytsov et al. addressed the problem of adding new data into the existing t-SNE environment using Local Inverse Distance Weighting Interpolation (LIDWI) without re-running. The outlier's handing is also addressed by LION-tSNE using outlier placement heuristic, which assumes that some percentile of outliers present in the designed t-SNE and determine the outliers from the newly added data points. In LION-tSNE, the sample t-SNE model is designed based on the random sample selection, which may cause the non representativeness of the data. The representative samples are selected by our earlier approach called $k$-NN sampling [19] and the resutlts are statistically significant which is measured by statistical method pairwise t-test [20].

This paper is an extension of our earlier paper presented in High Performance Computing, Data and Analytics (HiPC) [19], which deals with the preservation of the underlying cluster structure of high-dimensional data in low-dimensional t-SNE embedding with a representative sample. The underlying cluster structure preservation is measured in terms of a quantitative metric. In the existing methods, the low-dimensional embedding of t-SNE describes the quality of the structure preservation. Still, it is an open problem for giving the quantitative proof for the number of clusters that exist in the original data. The proposed novel representative $k$-NN sampling-based clustering approach for effective dimensionality reduction-based visualization finds the solution. In the first step, the proposed approach determines several distinct data samples using our earlier proposed $k$-NN sampling algorithm. The number of samples depends on the range of $\mathrm{k}$ (i.e., $1 \leq k \leq m$ ), which gives the neighborhood representation.

In the second step, the effective number of clusters existing in the original dataset is determined by the sampled data using clustering techniques such as $k$-Means [21], Agglomerative Hierarchical Clustering (AHC) [22], Balanced Iterative Reducing and Clustering using Hierarchies (BIRCH) [23], Fuzzy c-means (FCM) [24], Density-Based Spatial Clustering of Applications with Noise (DBSCAN) [25], Ordering Points To Identify the Clustering Structure (OPTICS) [26], Mean Shift (MS) [27], Spectral Clustering (SC) [28], Expectation-Maximization Gaussian Mixture Model (EMGMM) [29], Affinity Propagation (AP) [30] and Mini Batch $\mathrm{k}-$ Means (MBKM) [31]. Each clustering technique generates cluster labels for sampled data. The cluster labels of remaining data (i.e., data points other than selected samples) are labeled by the $k$-Nearest Neighbor $(k-\mathrm{NN})$ algorithm. The $k$-NN of each remaining data sample is subject to the selected samples. After assigning the labels to the remaining data samples, the representative sample of each clus- tering technique is determined by their external cluster validation index called Fowlkes-Mallows Index (FMI) [32]. In our contribution, we are also recommending the order sequence of clustering techniques among the selected techniques for a given dataset. We are also presenting the Compactness (CP) [33], Calinski-Harabaz Index (CHI) [33] and Contingency Matrix (CM) [34] of clustering techniques for gaining a more detailed analysis about clustering. The first technique in the order sequence denotes the best clustering technique and the representative samples of it considered as the samples for t-SNE model design for a given dataset. The cluster validation index provides the comparison between representative $k$-NN sampling-based clustering and the aggregate clustering (i.e., clustering on the whole dataset). The proposed representative $k$-NN sampling-based clustering is scalable to all clustering techniques which are suitable for numerical datasets. Also, we can apply any clustering technique to the large scale dynamic data with representative $k$-NN sampling-based clustering. Due to the paper limitation, we have chosen the most popular clustering techniques from different groups.

In the third step, the sample t-SNE model is designed on a representative sample of best clustering techniques, which transforms the high-dimensional data into low-dimensional embedding. The remaining data samples are added to the sample t-SNE environment with the help of LIDWI. The outliers from the remaining samples are identified and controlled by proposed heuristic and the identified outliers are placed into the t-SNE environment using outlier placement heuristic of Boystov et.al [17]. In the fourth step, the t-SNE embedding of input data is quantitatively evaluated by $k$-NN accuracy in the context of clustering and trustworthiness. The quantitative evaluation answers the question, how much structure of high-dimensional data is preserved by the low-dimensional $\mathrm{t}$-SNE embedding. The $k$-NN accuracy of $\mathrm{t}-\mathrm{SNE}$ embedding is measured in two ways: baseline accuracy and sampling accuracy. For quantitative performance evaluation, the $k$-NN accuracy of t-SNE embedding of representative $k$-NN sampling-based clustering and aggregate clustering results are compared with the ground truth class labels, which is measured in our earlier paper. In our earlier approach, we used ground-truth class labels for obtaining the $k$-NN accuracy of the t-SNE embedding but here we are using for checking the derived cluster purity of representative $k$-NN sampling-based clustering. The $k$-NN sampling-based clustering results and t-SNE embedding of it are analyzed by seven differently characterized toy and real-world datasets. In summary, our contribution consists of

- The order sequence of applicable clustering methods with representative $k$-NN sampling-based clustering using cluster validation index. The set of recommended techniques for the given dataset using a threshold. The comparison between representative $k$-NN sampling-based clustering and aggregate clustering results.

- The outliers from the remaining samples are identified and controlled by the proposed heuristic before adding into the $\mathrm{t}-\mathrm{SNE}$ embedding. The embedding structure quantitatively evaluated by $k$-NN accuracy in the context of clustering and trustworthiness.

The organization of the paper is as follows. In section 2, we present the background knowledge that gives the basic mathemati- 
cal intuition required for understanding the clustering techniques, t-SNE and other supplements. Section 3 presents the related work. In section 4, we present a detailed description of the proposed $k$-NN sampling-based clustering for the visualization of dynamic data. In section 5, we are providing the details of datasets, experimental setup and analysis of results. Finally, section 6 gives directions for future work and conclusions.

\section{Background Knowledge}

The following section describes the few techniques for understanding the background formulation of our work. Section 2.1 gives a detailed description of the selected clustering techniques for representative $k$-NN sampling-based clustering. Section 2.2 describes the mathematical intuition of the t-SNE algorithm. The intuition behind the addition of the inlier data point into an existing t-SNE environment is explained in section 2.3. In section 2.4. we are giving a detailed description of metrics that are used for the quantitative evaluation of embedding.

\subsection{Clustering and their performance measures}

Section 2.1.1 describes the selected clustering techniques which are used for representative $k$-NN sampling-based clustering. 2.1.2 provides detailed mathematical intuition of cluster validation indexes such as Fowlkes-Mallows Index (FMI), Compactness (CP), Calinski-Harabaz Index (CHI) and Contingency Matrix (CM).

\subsubsection{Clustering Techniques}

Clustering is unsupervised learning, where the similarly characterized data objects are grouped. The clusters of data objects can be represented as a set $\mathrm{C}$ of subsets $C_{1}, C_{2}, \ldots, C_{k}$ such that $\cap_{i=1}^{k} C_{i}=\phi$. The different clustering algorithms are proposed based on their measures of similarity: partitional, hierarchical, fuzzy theory-based, distribution-based, density-based, graph partition-based, grid-based, model-based and many more. The user decides the number of clusters present in the dataset by using a heuristic, trail-and-error and evolutionary approaches such as density and probability density. From the groups mentioned above, we have chosen the most frequently and popularly used algorithms for experimental evaluation. But proposed representative $k$-NN sampling-based clustering approach is scalable to all the clustering techniques which are suitable for the numerical datasets. We explored traditional clustering algorithm [35-37] for our work.

In partitional clustering, data points assigned to any one of the k-clusters using distance similarity measures such as Euclidean distance. k-means [21] clustering is one of the simplest, best-known and benchmarked partition-based clustering. The k-means clustering classifies the given data points through a user-defined number of clusters. The main goal of the k-means clustering is the initialization of an appropriate k-centroids, one for each cluster. The objective of $\mathrm{k}$-means is the minimization of the sum of square distance which can be defined as follows

$$
S S D=\sum_{j=1}^{k} \sum_{i=1}^{n}\left\|x_{i}^{(j)}-c_{j}\right\|^{2}
$$

where $\left\|x_{i}^{(j)}-c_{j}\right\|^{2}$ is a L2 norm between a data point $x_{i}^{(j)}$ and the cluster center $c_{j}$. In AP [30], at the initial stage, all data points are considered as centroids and nodes of the network. The clusters and their centroids are measured by transmitting the similarity message recursively. The Mini Batch k-Means (MBKM) [31] is an improved version of k-means, which performs clustering on batches instead of considering each point. Therefore, MBKM requires less computational time and is also applicable to large datasets.

In hierarchical clustering, groups are formed by iteratively dividing the data objects either in the bottom-up or top-down approach. The bottom-up approach is also known as Agglomerative Hierarchical Clustering (AHC) [22], in which initially each data object is considered as a separate group, then merging these small groups into larger and larger groups until a single group formation or certain threshold. The top-down approach is also known as Divisive Hierarchical Clustering (DHC), and it works in reverse order of the bottom-up approach. Both hierarchical approaches depend on the linkage criteria such as single, complete and average linkage. The linkage criteria determines the metric for merging two similar characterized small clusters. For example, single linkage criteria determines the minimum distance pair from the neighbor clusters $\min \left\{d(x, y): x \in C_{i}, y \in C_{j}\right\}$. The BIRCH [23] method has proposed to deal with large datasets, outliers in robust and also to reduce the computational complexity. The BIRCH method works on the idea of Cluster Features (CF) which is a height-balanced tree.

The basic idea of fuzzy theory-based clustering is that the discrete labeling is converted to continuous intervals, to describe the belonging relationship among objects more reasonably. The Fuzzy c-means (FCM) [24] is an extension of $k$-means where each data point can be a member of multiple clusters with membership value. The main advantage of FCM is that the formed groups are more realistic.

The density-based clustering finds the clusters based on the density of data points in a region. The principal objective of densitybased clustering is that for each instance of a group, there should be at least a minimum number of neighbor instances within the given radius. The DBSCAN [25] is the most well known density-based clustering. In DBSCAN clustering, the data objects fall into three groups: core-object, border-object and noise-object. The data points of the core-object group have enough number of neighbors in the given radius, and these data points are from the higher density region. The data points of the border-object group have fewer neighbors than the required number of neighbors in the given radius and they are in the neighborhood of the core object group. The data points of the noise-object group are not in either core or border-object group. The advantage of DBSCAN clustering is that the generated clusters are in arbitrary shape based on the given parameters such as radius and number of minimum instances. The improved version of DBSCAN is OPTICS [26], which overcomes the limitations of DBSCAN. Mean-shift [27] algorithm determines the mean of offset of the current data point, the next point is identified based on the current point and the offset. The process will continue until some criteria is satisfied.

The spectral clustering [28] is a graph partition-based technique in which clustering is obtained by similarity graph partition. The paper [28] describes the different ways of constructing the similarity graph. While building the similarity graph, the data objects are con- 
sidered as vertices and the similarity (i.e., the similarity distance) between the data objects is a weighted edge. The critical idea of similarity measure is to find a method of graph partition by making edges between different groups have low edge weight and the edges of the same group have more edge weights. In similarity graph partitioning, the cluster analysis is carried out by feature space obtained by the eigenvectors corresponding to $\mathrm{k}$ largest eigenvalues of the laplacian matrix [38] of similarity graph. The spectral clustering is suitable for the datasets with arbitrary shape, high-dimensions and outliers.

The EMGMM [29] is a model as well as a distribution-based clustering technique in which cluster data obey the same independent Gaussian distribution which exists in the original data distribution. The generated clusters are more realistic because a probability distribution obtains them.

The performance and applicability of clustering depends on the used dataset characteristics. Therefore, the researchers empirically state that none of the clustering technique is generalized for any datasets.

\subsubsection{Cluster validation Measures}

In our experimentation, we have used two internal and one external cluster validation measure to evaluate the performance of clustering techniques. The internal measure computes the performance of clustering without using the ground-truth class labels. The compactness (CP) [33] is an internal cluster validation measure which can be computed as follows

$$
C P=\frac{1}{n} \sum_{l=1}^{k} n_{l}\left(\frac{\sum_{x_{i}, x_{j} \in C_{l}} d\left(x_{i}, x_{j}\right)}{n_{l}\left(n_{l}-1\right) / 2}\right)
$$

where $d\left(x_{i}, x_{j}\right)$ is Euclidean distance between two objects in cluster $C_{l}$ and $n_{l}$ is the number of objects in $C_{l}$. The smaller CP value of clustering is more compact and gives better clustering results. The Calinski-Harabaz Index (CHI) [33] or Variance Ratio Criterion is defined as the ratio of between-clusters dispersion mean and the within-cluster dispersion mean. The $\mathrm{CHI}$ is an internal cluster validation measure which is given by

$$
C H I=\frac{\sum_{l=1}^{k} n_{l} d^{2}\left(C_{l}, C\right) /(N C-1)}{\sum_{l=1}^{k} \sum_{x \in C_{l}} d^{2}\left(x, C_{l}\right) /(n-N C)}
$$

Where $n$ is the dataset size, $C$ is the dataset center, $n_{l}$ is the size of $l^{t h}$ cluster, $C_{l}$ is the $l^{\text {th }}$ cluster centroid and $\mathrm{NC}$ is the number of clusters.

The external validation measure knows the ground truth class labels. The primary purpose of the external validation index is to choose an optimal clustering algorithm for a given dataset. The external validation measure also checks the cluster purity. In our experiment, we used Fowlkes-Mallows Index (FMI) [32] is the geometric mean between precision and recall. The FMI defined as follows

$$
F M I=\frac{T P}{\sqrt{(T P+F P)(T P+F N)}}
$$

The FMI value is bounded between 0 and 1,1 denotes that the obtained clusters and the given ground truth classes are the same. The large FMI indicates that the obtained clustering is purer.
We also measured the contingency matrix that reports the intersection cardinality for every true/predicted cluster pair. The contingency matrix gives sufficient statistics for all clustering metrics. But it is hard to interpret the contingency matrix of extensive clustered data.

\subsection{Student t-Distributed Stochastic Neighbor Embed- $\operatorname{ding}(t-S N E)$}

The t-SNE [9] algorithm introduced by Laurens Van Der Maaten and Geoffrey Hinton in 2008, based on the SNE algorithm. The principal objective of SNE is to preserve the underlying structure of high-dimensional data in low-dimensional embedding space. Lets assume the given input data $\mathcal{X}=\left\{x_{1}, x_{2}, \ldots . ., x_{N}\right\}$ where each $x_{i} \in \mathbb{R}^{D}$ is a D-dimensional vector. The t-SNE computes the embedding $\mathcal{Y}=\left\{y_{1}, y_{2}, \ldots, y_{N}\right\}$ of $\mathcal{X}$ where each $y_{i} \in \mathbb{R}^{d}$ is a d-dimensional vector, where $d \ll D$ and most commonly $\mathrm{d}=2$ or 3 . The similarity between $x_{i}$ and $x_{j}$ of $\mathcal{X}$ is calculated by conditional probability $p_{i j}$ is given by

$$
p_{j / i}=\frac{\exp \left(\frac{-d\left(x_{i}, x_{j}\right)^{2}}{2 \sigma_{i}^{2}}\right)}{\sum_{k \neq i}^{N} \exp \left(\frac{-d\left(x_{i}, x_{k}\right)^{2}}{2 \sigma_{i}{ }^{2}}\right)}, p_{i / i}=0, p_{i j}=\frac{p_{j / i}+p_{i / j}}{2 N}
$$

where the bandwidth $\sigma_{i}$ of Gaussian kernel, is obtained by binary search by matching the perplexity of $P_{i}$ and the user-defined perplexity $(\mu)$ which is given as a parameter. The perplexity is a smooth measure of an adequate number of neighbors for each data point. The equality and perplexity of $P_{i}$ is as follows

$$
\mu=2^{H\left(P_{i}\right)} \text { where } H\left(P_{i}\right)=-\sum_{j}^{N} p_{j / i} \log _{2} p_{j / i}
$$

where $H\left(P_{i}\right)$ denotes the entropy and $P_{i}$ is the conditional probability distribution across all data points for the given $x_{i}$. The $y_{i}$ and $y_{j}$ are the corresponding low-dimensional values of $x_{i}$ and $x_{j}$ (i.e., the values of $\mathcal{Y}$ are initialized by Gaussian or uniform distribution). The similarity between $y_{i}$ and $y_{j}$ is defined as

$$
q_{i j}=\frac{\left(1+d\left(y_{i}, y_{j}\right)^{2}\right)^{-1}}{\sum_{l}^{N} \sum_{k \neq l}^{N}\left(1+d\left(y_{l}, y_{k}\right)^{2}\right)^{-1}}
$$

where $d\left(y_{i}, y_{j}\right)$ is defined as distance similarity measure such as Euclidean distance. In low-dimensional embedding, the similarity $q_{i j}$ is obtained by student t-distribution with one degree of freedom. But, the similarity $p_{i j}$ of high-dimensional data uses the Gaussian distribution. The cumulative function curvature of Gaussian distribution is flatter than the cumulative function curvature of student t-distribution with one degree of freedom. The principal idea of using student t-distribution in low-dimensional embedding is to overcome the crowding problem [7].

If $p_{i j} \sim q_{i j}, \forall_{i \neq j} \in N$, then the given data is perfectly embedded into the low-dimensional space. Otherwise, compute the KL-divergence (i.e., error) between $p_{i j}$ and $q_{i j}$ that is equal to the cross-entropy in Information Retrieval System (IRS). The cost function $(\mathbf{C})$ or objective of t-SNE is defined as follows

$$
\mathbf{C}=K L(P \| Q)=\sum_{j}^{N} p_{i j} \log \frac{p_{i j}}{q_{i j}}
$$


The simple gradient descent applied on cost function $\mathbf{C}$ for obtaining the optimization or minimization of it. The simple gradient descent of cost function $\mathbf{C}$ given by

$$
\frac{\partial \mathbf{C}}{\partial y_{i}}=4 \sum_{j \neq i}\left(p_{i j}-q_{i j}\right) q_{i j} Z\left(y_{i}-y_{j}\right)
$$

where $Z=\left(1+d\left(y_{i}, y_{j}\right)^{2}\right)^{-1}$ is a normalization term of student tdistribution. The Equation 9 produce either positive or negative values depending on the $p_{i j}$ value. If an Equation 9 gives positive value then there is an attractive force among the $y_{i}$ and $y_{j}$ of embedding space. Otherwise, there is a repulsive force among the $y_{i}$ and $y_{j}$ of embedding space. The degree of repulsion depends solely on the closeness of points in the embedding space. In the optimization process, the early exaggeration coefficient $\alpha>1$ plays a paramount role in forming groups of similar objects of high-dimensional data in low-dimensional embedding space. In the early exaggeration process, the elements of similarity matrix (P) (i.e., $p_{i j}$ 's) multiplied by the early exaggeration coefficient, which is measured by the intuition given by George C. Linderman and Stefan Steinerberger [39]. Therefore, similar data objects bring near to each other in low-dimensional embedding space. This process can happen at the early stage of optimization. The gradient descent of $\mathbf{C}$ after early exaggeration is

$$
\frac{\partial \mathbf{C}}{\partial y_{i}}=4 \sum_{j \neq i} \alpha p_{i j} q_{i j} Z\left(y_{i}-y_{j}\right)-4 \sum_{j \neq i} q_{i j}^{2} Z\left(y_{i}-y_{j}\right)
$$

The momentum and learning rate parameters improve the optimization process of the cost function. The momentum parameter reduces the number of iterations of the cost function optimization. At the initial stage of iteration, the momentum value is small until the map points have become moderately well organized. The optimization is improved by input approximation and tree-based algorithms [13], which reduce the memory and computational complexity. The updated values of $\boldsymbol{Y}$ at iteration $t$ is obtained by

$$
\boldsymbol{y}^{(t+1)}=\boldsymbol{y}^{(t)}+\eta \frac{\partial \mathbf{C}}{\partial y_{i}}+\alpha(t)\left(\boldsymbol{y}^{(t-1)}-\boldsymbol{y}^{(t-2)}\right)
$$

where $\alpha(t)$ is momentum at $t^{\text {th }}$ iteration, $\eta$ is learning rate. The work-flow of t-SNE is shown in the Figure 1 .

The well-separated clusters of input data are well preserved in the low-dimensional embedding by setting the early exaggeration coefficient $\alpha$ and learning rate $\eta$. The intuition for setting the above parameter is derived by George C. Linderman and Stefan Steinerberger in [39]. According to the George C. Linderman and Stefan Steinerberger observations the early exaggeration coefficient $\alpha$, learning rate $\eta$, and minimum probability $p_{i j}$ 's of same cluster objects(i.e., $x_{i}$ and $x_{j}$ belong into the cluster $C_{l}$ for all $i \neq j$ ) is defined as follows.

$$
\alpha \sim \frac{n}{10}, \eta \sim 1 \text { and } p_{i j}=\frac{1}{10 n\left|\pi^{-1}(\pi(i))\right|}
$$

where $\pi:\{1,2, \ldots ., n\} \rightarrow\{1,2, \ldots, k\}$ assigns each data point to one of the $\mathrm{k}$ clusters.

\subsection{Local Inverse Distance Weighting Interpolation (LIDWI)}

The LIDWI [40] maps new data point $x \in \mathcal{R}^{D}$ into the existing embedding $y_{i} \in \mathcal{R}^{d}$, where $\{i=1,2, \ldots \ldots . . m\}$. The LIDWI determines the value of $x$ as a weighted sum of values $y_{i}$, where weight is proportional to inverse distances. The LIDWI of $x$ is

$$
\operatorname{LIDWI}(x)=\sum_{\left\|x-x_{i}\right\| \leq r_{x}} w_{i}(x) \cdot y_{i}, w_{i}(x)=\frac{\left\|x-x_{i}\right\|^{-p}}{\sum_{\left\|x-x_{i}\right\| \leq r_{x}}\left\|x-x_{i}\right\|^{-p}}
$$

for instance, when the data point $x \rightarrow x_{i}$, the inverse distance $\left\|x-x_{i}\right\|^{-1} \rightarrow \infty$, the corresponding weight $w_{i}(x) \rightarrow 1$ (i.e., $\forall_{j \neq i} w_{j}(x) \rightarrow 0$ due to the normalization) and $\operatorname{LIDWI}(x) \rightarrow y_{i}$. The neighbor points selection is obtained by a radius $r_{x}$ parameter. The parameter $r_{x}$ value is calculated by the heuristic proposed by Andrey Boytsov et.al. [17]. In LIDWI, the power parameter $p$ plays an important role. For instance, very small value of $p$ predicts the value of $x$ around the center: $y \approx \operatorname{mean}\left(y_{i}\right)$ (unless $x=x_{i}$ ) even the distance $\left\|x-x_{i}\right\|$ is low because the weight distribution is close to uniform. When the power parameter is high and the distance $\left\|x-x_{i}\right\|$ is low, the weight $w_{i}(x)$ of very first nearest neighbor is dominating all other neighbors, therefore $y \approx y_{i}$ where $i=\operatorname{argmin}\left\|x-x_{j}\right\|$. The overfitting suffers from either too small or too large values of power parameter $p$. In LOIN-tSNE, the authors proposed a generalization for obtaining power parameter by using leave-one-out cross-validation of the training sample. The computation of the generalized power parameter is obtained by applying the LIDWI for each training sample that produces the estimation of each $y_{i}$. Then the mean square distance between the estimated $y_{i}$ 's and real $y_{i}$ 's is computed. The optimal power parameter is obtained by optimizing the mean square error (i.e., the mean square distance is minimum). The obtained power parameter is considered as a metric. However, this metric is heuristic, not an exact criterion.

\subsection{Performance evaluation metrics}

\subsection{1 k-NN accuracy}

The existence of the cluster structure of high-dimensional data in low-dimensional embedding is quantitatively measured by $k$-NN accuracy of the t-SNE embedding in the context of clustering. The $k$-NN accuracy is defined as the percentage of the neighbors having the cluster label equivalent to the observational point cluster label.

\subsubsection{Trustworthiness}

Trustworthiness [41] is one of the measure to evaluate the quality of the t-SNE embedding. Trustworthiness is defined as any unexpected nearest neighbors in the output space are penalized in proportion to their rank in the input space.

$$
T(k)=1-\frac{2}{N k(2 N-3 k-1)} \sum_{i=1}^{n} \sum_{x_{j} \in U_{k}\left(x_{i}\right)}\left(r\left(x_{i}, x_{j}\right)-k\right)
$$

where $U_{k}\left(x_{i}\right)$ is the $k$-NN of $x_{i}$ in embedding space, $r\left(x_{i}, x_{j}\right), i \neq j$ the rank of $x_{j}$ when the data vectors are ordered based on their 


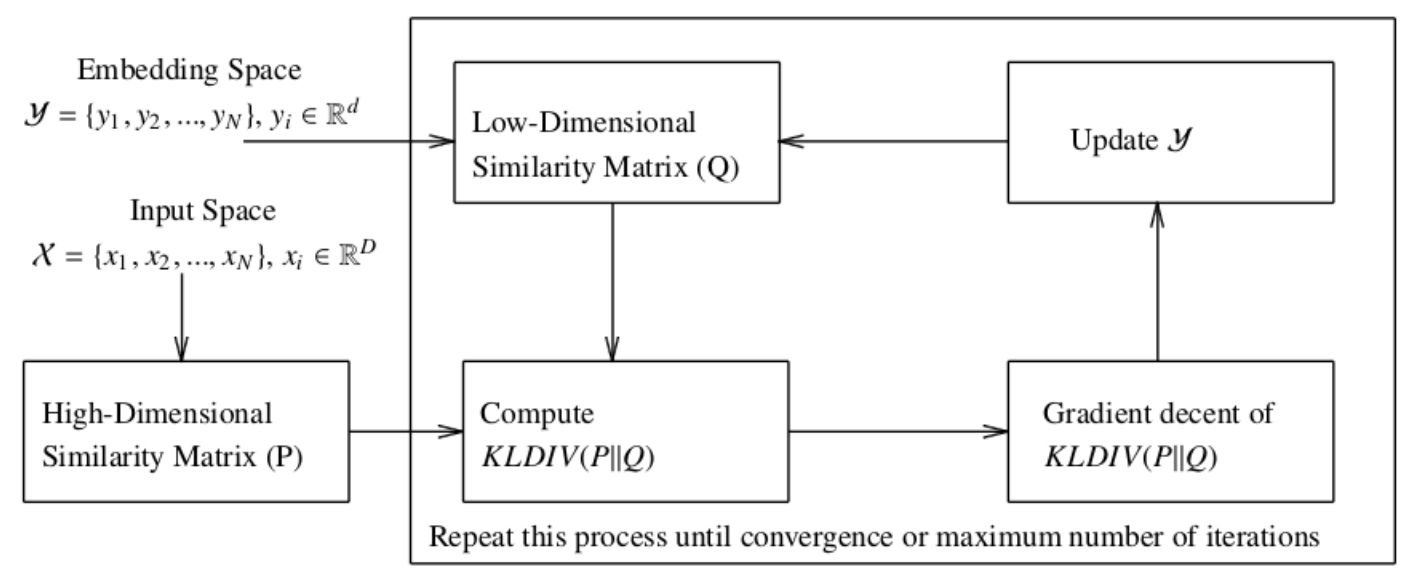

Figure 1: Work-flow of t-SNE algorithm [19]

Euclidean distance from the data vector $x_{i}$ in the original data space. It is bounded between 0 and 1 , where 1 represents the complete structure of the data preserved in the embedding space.

\section{Related Work}

Our earlier proposed $k$-NN sampling based tSNE mainly concentrates on the preservation of the underlying structure of highdimensional data in low-dimensional embedding space with representative samples. The obtained low-dimensional embedding structure describes the quality of the structure using ground-truth class labels. But the low-dimensional embedding does not give the quantitative proof for the number of clusters that exist in the original data. In [39] authors gave the theoretical observations for well-separated clusters of high-dimensional data in low dimensional embedding space.

The researchers have proposed various methods to incorporate new data samples into the existing t-SNE environment. Most of the existing techniques designed a mapping function $f: \mathcal{X} \rightarrow \mathcal{Y}$, which accepts multi-dimensional data and returns its low-dimensional embedding. The designed mapping functions are used for incorporating the new data samples into the existing t-SNE environment. In [14-16] authors have proposed different approaches for adding a new data sample or scaling up the t-SNE algorithm.

Andrey Boytsov et.al. [17] proposed the LION-tSNE algorithm based on local IDWI for adding new data sample into an existing tSNE environment. It also addresses the outlier handling approaches. Our earlier work extended the idea of the LION-tSNE algorithm by proposing a $k$-NN sampling method for designing a representative sampling based t-SNE model. It allows the selection of the sample concerning their k-nearest neighbors instead of random sampling. In this paper, we are proposing the novel representative $k$-NN sampling-based cluster approach for effective dimensionality reduction-based visualization of dynamic data, which determines the underlying cluster by using the most popular clustering techniques. The obtained cluster structure is quantitatively evaluated by $k$-NN accuracy in the context of clustering and trustworthiness.

\section{Proposed representative $k$-NN sampling- based clustering for effective visualiza- tion Framework}

The proposed representative $k$-NN sampling-based clustering for effective dimensionality reduction-based visualization of dynamic data framework is shown in the Figure 2 It has four phases: at phase 1, data preprocessing is done for removing the redundant data points and filling the empty variables with appropriate values. At phase 2, the proposed $k$-NN sampling-based clustering is applied to determine: (i) The generation of $k$ distinct samples using mutual $k$-NN sampling with static graph updation algorithm. (ii) The list of clustering techniques applicable for the given dataset. (iii) The optimal sample size produces the best grouping for each concerned clustering technique. (iv) The ordered sequence of recommended clustering techniques for the given dataset from which the best clustering technique is selected by using FMI scores. The detailed representative $k$-NN sampling-based is clustering presented in section 4.1. At phase 3, the dimensionality reduction-based visualization is obtained by t-SNE algorithm, which derives the low-dimensional embedding of data and the LIDWI algorithm is used to interpolate the new data points into the learned t-SNE model. The outliers from the remaining data points are identified and controlled by the proposed heuristic. The dimensionality reduction-based visualization is described in section 4.2 In the final phase, the quantitative measure of the t-SNE embedding is computed by $k$-NN accuracy and trustworthiness. The process of quantitative metric derivation is described in section 4.3

\subsection{Representative $k$-NN sampling-based clustering}

Initially, the $k$ distinct samples are generated by the modified algorithm of our earlier proposed method. The sample generation depends on the parameter $k$ of $k$-NN sampling. Each $k$ generates one distinct sample; the boundary of $k$ is denoted as $1 \leq k \leq m$, where $m$ is the maximum number of neighbors required for preserving the behaviour of any data point of the given dataset. Our earlier approach has given two different $k$-NN sampling strategies 


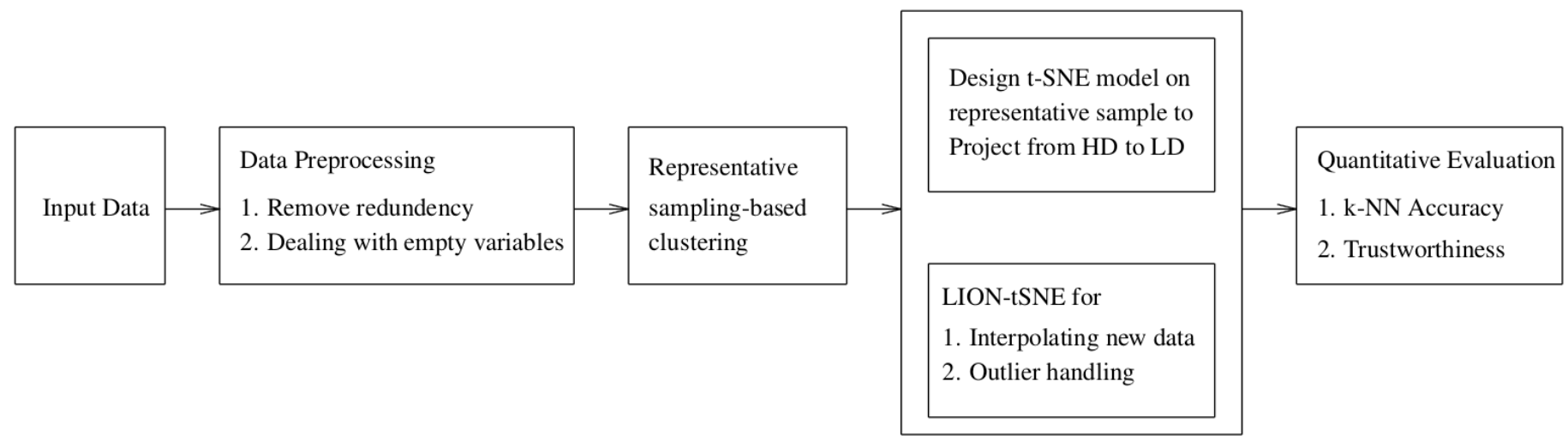

Figure 2: Proposed framework of representative $k$-NN sampling-based clustering for an effective dimensionality reduction-based visualization of dynamic data using LION-tSNE with quantitative measure of cluster accuracy

such as static and dynamic $k$-NN sampling based on $k$-NN graph modification. In this paper, we are considering only static $k$-NN sampling in which the $k$-NN graph is modified statically that means no new edge is added among any two vertices of the $k$-NN graph. The main reason for selecting static $k$-NN sampling is that the t-SNE embedding results of it are more statistically significant than the dynamic one. The Nearest Neighbor score (NN_score) and Mutual Nearest Neighbor score (MNN_score) of each node of the $k$-NN graph are used for selecting the samples. Lets assume $k$-NN graph is a directed graph $G=(V, E)$, the edge $E\left(v_{1}, v_{2}\right)$ gives $v_{2}$ as a neighbor of $v_{1}$ and neighborhood of $v_{1}$ is denoted by $N_{v 1}$. The out-degree of each vertex is equal to $k$, and the in-degree of a vertex depends on the neighborhood property of other vertices (i.e., the data point $x_{i}$ belong into the neighborhood of other datapoints). In our method, each data point is a vertex of the $k$-NN graph and $k$ is a parameter for deriving the edges between neighboring vertices. The NN_score of data point $x_{i}$ is equal to the in-degree of $x_{i}$ which is defined as follows

$$
N_{-} \operatorname{score}\left(x_{i}\right)=\left|\left\{x_{j} \mid x_{i} \in N_{x_{j}}\right\}\right|, \forall_{j \neq i} x_{j} \in \mathcal{X}
$$

where $\mathcal{X}$ denotes whole data set, $N_{x_{j}}$ denotes the neighborhood of $x_{j}$. The MNN_score of data point $x_{i}$ is at most $k$ which is given by

$$
M N N_{-} \operatorname{score}\left(x_{i}\right)=\left|\left\{x_{j} \mid x_{i} \in N_{x_{j}}\right\}\right| \text { where } x_{j} \in N_{x_{i}}, \forall_{j \neq i} x_{j} \in \mathcal{X}
$$

From the NN_score and MNN_score matrix the Representative Sample (RS) $x_{i}$ selection and the sample $x_{j}$ Represented by Representative Sample (RRS) $x_{i}$ is given by

$$
\begin{aligned}
& R S\left(x_{i}\right)=\text { first_index }\left\{\operatorname{argmax}_{x_{i} \in \mathcal{X}}\left\{N_{N_{-}} \operatorname{score}\left(x_{i}\right)\right\} \cap\right. \\
& \left.\qquad \operatorname{argmax}_{x_{i} \in \mathcal{X}}\left\{M N N_{-} \operatorname{score}\left(x_{i}\right)\right\}\right\}
\end{aligned}
$$

$$
R R S\left(x_{j}\right)=\left\{x_{j} \mid x_{j} \in N_{x_{i}}\right\} \text { where } x_{i} \in N_{x_{j}}, \forall_{j \neq i} x_{j}, x_{i} \in \mathcal{X}
$$

The updated mutual $k$-NN sampling with a static graph updation algorithm is shown in Algorithm [1 Initially, the Train_samples (i.e., representative samples set) and Rep_samples (i.e., the sample represented by the selected training sample that is the first nearest mutual neighbor of train sample) are null sets. For each iteration, the data point $\mathrm{x}$ which has maximum $\mathrm{NN}$ and maximum MNN score appended to the Train_samples set. The data point $y$ is appended to the Rep_samples if $y \in N_{x}$ and $x \in N_{y}$ that denotes the point $x$ and $y$ are more similar (i.e., the distance $d(x, y)<d(x, z)$ where $\left.\forall z \in N_{x}\right)$ to each other. The data point $x$ and $y$ are deleted from $X$ and their corresponding vertices are deleted from the $k$-NN graph. After deleting the vertices of $x$ and $y$, the corresponding in-edges and out-edges are removed from the $k$-NN graph and the graph is updated accordingly. The elements of Train_samples and Rep_samples are obtained iteratively, the iteration repeats until the NN_score of the remaining $X$ is equal to zero.

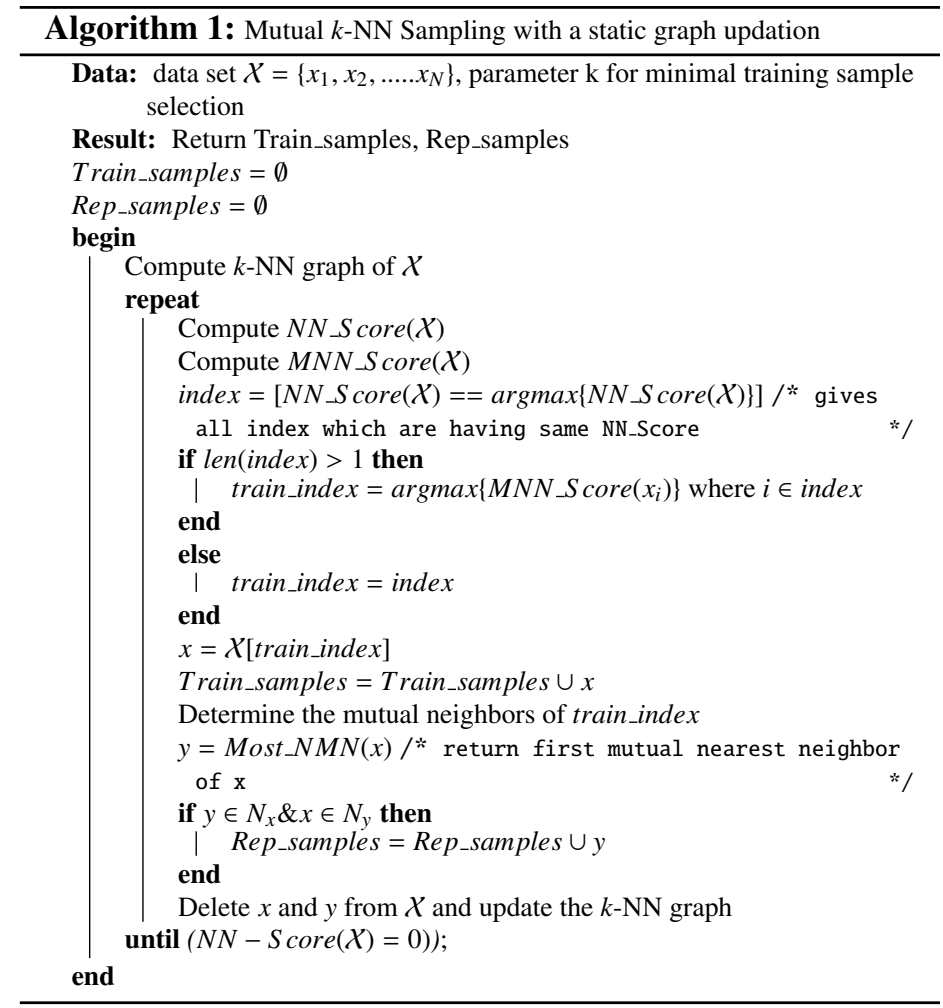

In our earlier approach of static graph updation, we have considered the whole mutual neighborhood set of $x$ as the samples represented by $x$. The whole neighborhood set selection causes 
loss of information due to that reason the earlier approach select Train_samples set at an early stage of $k$ value. The data points of the remaining $\mathcal{X}$ (i.e., the data point do not belong into either Train_samples set or Rep_samples set) is handled in two different ways. Handling of the remaining samples is described in subsection 4.4. The mutual $k$-NN sampling with a static graph updation algorithm generates a various set of Train_samples and Rep_samples with different $\mathrm{k}$-values.

The clustering techniques which are described in subsection 2.1.1 are applied on each Train_samples set of mutual $k$-NN sampling with a static graph updation algorithm that is called a samplingbased clustering. The cluster labels for the data points other than the Train_samples are assigned by using $k$-NN algorithm. For example, the dataset size is $N$, the Train_samples size is $n$ and then the unmarked sample size is $N-n$ (i.e., the samples are not assigned with any labels). The $k$-NN of each unmarked sample is derived from the associated Train_samples set. The label assignment of each unmarked sample depends on the labels of its $k$-NN, a label with maximum occurrence in $k-\mathrm{NN}$ that is assigned to a sample. From each clustering technique, the optimal Train_samples set is obtained by the FMI score. The FMI is an external validation index that uses the ground truth class labels. Therefore, the number of clusters is defined as a constant that is equal to the number of ground truth classes. The FMI score of optimal Train_samples of clustering techniques generates an order sequence of the clustering techniques. From the order sequence, we can recommend the most desirable techniques for a given dataset from the selected set of techniques. From this recommendation, the best suitable technique is selected and its optimal Train_samples set is considered as the best representative sample. The threshold parameter is used to derive the recommended techniques, which is defined as the FMI score difference between two adjacent techniques of order sequence. The three cluster validation index such as FMI score, CHI score and $\mathrm{CP}$ of representative $k$-NN sampling-based clustering are compared with their aggregate clustering (i.e., clustering on whole dataset) validation index of chosen clustering techniques. The result comparison is discussed in subsection 5.3. The algorithm of representative $k$-NN sampling-based clustering is shown in Algorithm 2 . The embedding of selected optimal Train_samples and addition of other data samples into an existing t-SNE environment is described in the following section.

\subsection{Dimensionality reduction-based visualization}

The subsection 4.2.1 describes the low-dimensional embedding of a representative sample with t-SNE algorithm. Subsection 4.2.2 describes the addition of new data samples into an existing t-SNE environment that is called out-off-sample extension.

\subsubsection{Low-dimensional embedding of a representative sample with t-SNE algorithm}

Barnes-Hut t-SNE (BH-tSNE) [13] algorithm is an optimized version of the t-SNE algorithm. BH-tSNE optimizes the t-SNE objective function by input similarity approximation and gradient descent approximation. Therefore, it generates low-dimensional embedding of data with minimum computational and memory complexity than original t-SNE. In our approach, the BH-tSNE algorithm is used in two different ways for calculating the low-dimensional embedding space: 1. Baseline t-SNE embedding, 2. Sampled t-SNE embedding. The baseline t-SNE embedding is obtained by applying BH-tSNE on the whole dataset. In contrast, the sampled t-SNE embedding is obtained by applying BH-tSNE on the best representative sample, which is selected from the representative $k$-NN sampling-based clustering. The Baseline t-SNE embedding results analyze the overall structure of the data in low-dimensional embedding. The sampled t-SNE embedding results analyze the data structure with sampled data and allows the addition of new data samples into an existing t-SNE environment, which solves the scalability issue of the t-SNE. For obtaining a well-separated cluster in low-dimensional t-SNE embedding, the value of early exaggeration coefficient $\alpha$, learning rate $\eta$ and input similarity probability $p_{i j}$ 's are adjusted according to the George C.linderman and Stefan Steinerberger intuition. In our experimentation, the initial solutions of t-SNE is assigned in three different ways, such as random, PCA based and MDS based initial solutions. The random initial solution takes many iterations for convergence. The PCA and MDS based initial solutions overcome the problem of random initialization and they produce better accuracy results, but their initial solution is cost-effective. Adding new data points into a designed t-SNE model is discussed in the below section.

\subsubsection{Out-off-sample extension: Interpolation and Outlier han- dling}

The addition of new data point to t-SNE embedding depends on the parameter $r_{x}, r_{y}$ and $r_{\text {close }}$ values. The value of parameter $r_{x}, r_{y}$ and $r_{\text {close }}$ is obtained from the best representative sample and the t-SNE embedding of it. The parameter $r_{x}$ is defined as the percentile of the 1-NN distance of a representative sample that decides whether the given new data point is either inlier or outlier. In our proposal, we came to know that the objects of the representative sample set are representing at least one sample of the dataset. Therefore, the representative sample set does not contain any outlier object and we have considered the parameter $r_{x}$ as the maximum $1 \mathrm{NN}$ distance of it. If the new data point $x$ has at least one data point within the $r_{x}$ from the representative sample set, then $x$ is an inlier, otherwise outlier. The dilation factor (df) is used to derive a heuristic $r_{x}=(1+d f) * r_{x}$ which controls the consideration of outliers percentage. The LIDWI interpolation technique is used for adding an inlier data point to the t-SNE embedding of the representative sample set. The outlier placement depends on the parameter $r_{y}$ and $r_{\text {close }}$. The outliers placed into the t-SNE embedding of the representative sample set according to the heuristic of Boytsov et.al. [17]. The $k$-NN accuracy and trustworthiness of t-SNE embedding quantitatively evaluate the existence of a high-dimensional cluster in the low-dimensional embedding. The quantitative evaluation described in the following section.

\subsection{Quantitative metric derivation: $k$-NN accuracy}

In our experimentation, the $k$-NN accuracy is calculated in two different ways: baseline accuracy and sampling accuracy. The $k$ $\mathrm{NN}$ accuracy of t-SNE embedding of aggregate data is known as 


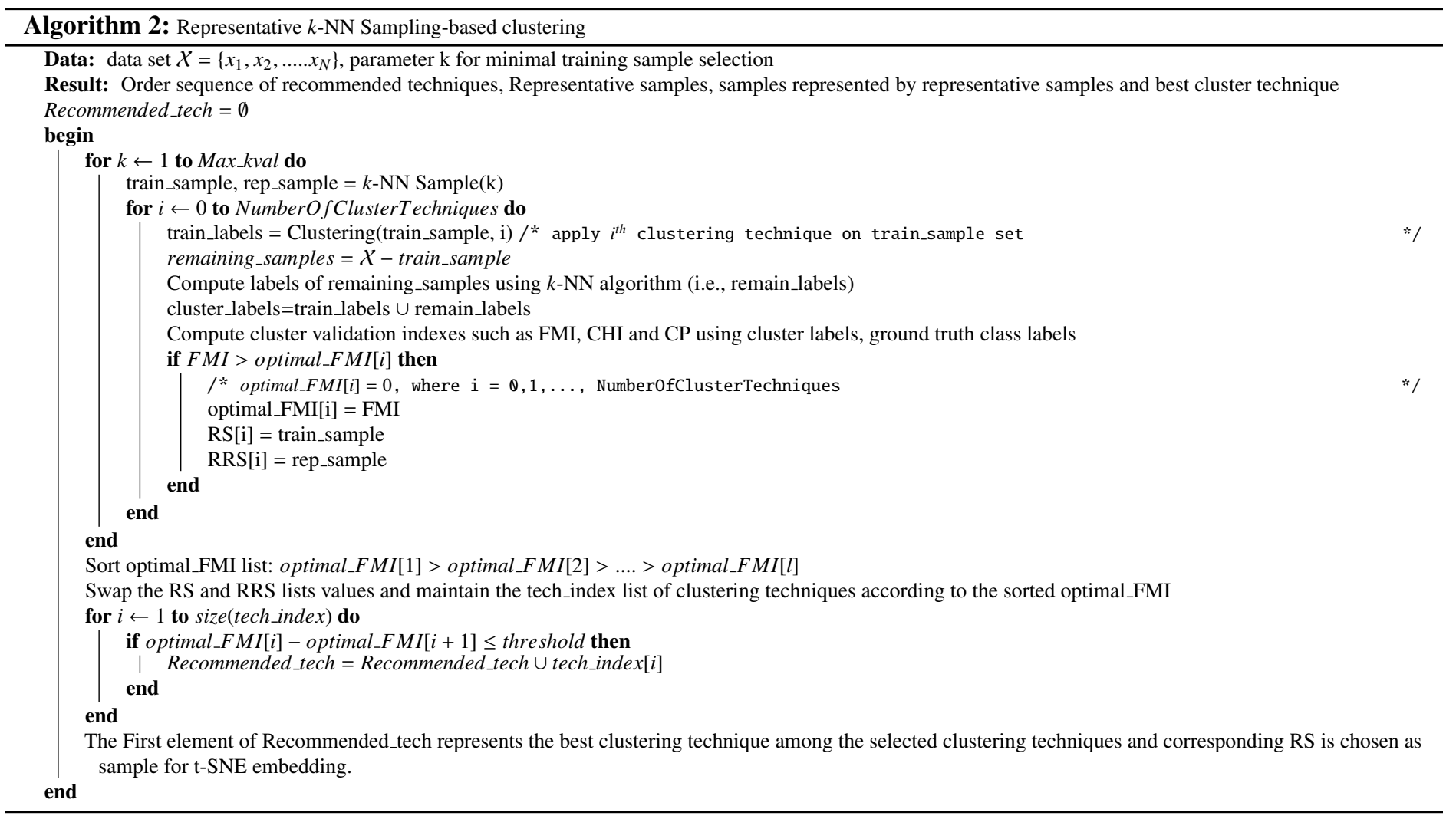

baseline accuracy. The sampling accuracy is computed in three different forms such as training accuracy, test accuracy and overall accuracy. The $k$-NN accuracy of t-SNE embedding of the representative sample is known as training accuracy. The $k$-NN accuracy of interpolated samples (i.e., the samples other than the representative sample) is known as test accuracy. The $k$-NN accuracy of combined low-dimensional space (i.e., the integration of t-SNE embedding of sampled data and interpolated data) is known as overall accuracy. The $k$-NN accuracy typically depends on the parameter $k$, which is considered as fixed in our experimentation which is discussed in subsection 5.2. For instance, the smaller $k$ will give good performance accuracy and while increasing the $k$, performance accuracy will decrease. The selection of parameter $k$ also plays a paramount role in $k$-NN accuracy measure. The relationship between the parameter $\mathrm{k}$ and accuracy is shown in the Figure 3 . For quantitative performance evaluation, the $k$-NN accuracy of representative $k$-NN samplingbased clustering and aggregate clustering are compared with the $k$-NN accuracy of ground truth class labels, which is discussed in subsection 5.3 .

\section{4 t-SNE sample selection criteria}

In our experimentation, we have considered two different sample selection criteria for designing the t-SNE model. In the first criteria, the samples are mostly representative of at least one or more other samples. The samples are obtained by a mutual $k$-NN sampling with a static graph updation algorithm and the best representative sample is selected by the representative $k$-NN sampling-based clustering. We observe that most of these samples were chosen from the dense region because they produce good NN_score and MNN_score.
Therefore, these samples do not contain any outliers. In this criterion, the outliers from the remaining sample addition are not sufficiently identified by the adequate $1-\mathrm{NN}$ distance. The outliers consideration is controlled by the proposed heuristic, which is discussed in subsection 4.2.2 In the second criteria, the data points added to the representative sample from the remaining data points using $(t, m, s)$-Nets sampling [42]. For the addition of $(t, m, s)$-Nets samples, we used the same procedure of our earlier work. The $(t, m, s)$-Nets select samples randomly in a uniform distribution. These samples may not represent any other samples and the samples may change among the executions due to the randomness. Therefore, the samples of this criteria may contain outliers. The outliers of this criteria are handled in similar ways as they are handled in our earlier approach.

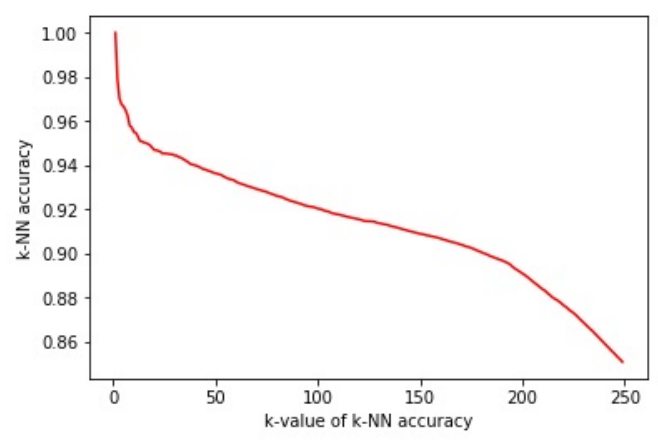

Figure 3: Relationship between k-value of $k$-NN accuracy and $k$-NN accuracy which is obtained from the baseline accuracy of $k$-NN sampling-based clustering. 


\section{Experimental result analysis}

Here we are briefing the datasets, experimental setup and the result analysis. Section 5.1 describes the numerical datasets of different characterizations. The details about the experimental setup is given in section 5.2 The results analysis of each dataset is described in section 5.3 .

\subsection{Datasets}

In our experimental evaluation, we have considered differently characterized datasets such as IRIS, Breast-Cancer, Leukemia, Wine, MNIST, Olivetti-Faces, and COIL-20. The Table 1 provides a detailed description of all the datasets which are downloaded from the UCI Machine Learning repository [43]. For our experimentation, the features of IRIS, Breast-Cancer and Wine datasets are normalized between zero and one, which improves the computational complexity. Initially, the KernelPCA [44] is applied to reduce the dimensionality of the high-dimensional datasets such as Leukemia, MNIST, Olivetti-Faces and COIL-20. The Leukemia dataset is a micro-array of gene expression. The MNIST, Olivetti-Faces and COIL-20 are image datasets that are represented in pixel orientations. The MNIST is a handwritten digits dataset. The Olivettifaces dataset consists ten face images of 40 individuals with small variation in viewpoint, the addition of glass and large variation in expression. The COIL-20 is an image of 20 group objects such as animals, furniture and etc.

Table 1: Overview of datasets along with their size, dimensions, and classes

\begin{tabular}{lccc}
\hline Dataset Name & Size & Dimensions & \# Classes \\
\hline IRIS & 150 & 4 & 3 \\
Breast Cancer & 569 & 30 & 2 \\
Leukemia-ALL-AML & 72 & 7129 & 2 \\
Wine & 178 & 13 & 3 \\
MNSIT & $70 \mathrm{~K}$ & 784 & 10 \\
Olivetti faces & 400 & 10304 & 40 \\
COIL-20 & 1440 & 1024 & 20 \\
\hline
\end{tabular}

\subsection{Experimental configuration}

In our experimentation, The parameter $k$ of Algorithm 1 is initialy considered as $1 \leq k \leq 50$. The upperbound of $k$ is equal to largest perplexity value from the literature study [9]. The perplexity is set between 5 and 50 for a fairly good visual representation of any real-world data. In our proposal, the samples of any clustering technique depends on the parameter $k$. Also, we observed that when there is an increment in parameter $k$ then there is an increment or no change in FMI score of clustering until certain $k$ value. The FMI score becomes stable afterwards. In our experimentation at most of the cases, the selected clustering technique generates representative sample with $k$ value less than or equal to 20 . In other cases, the cluster technique generates representative sample with $k$ value greater than 20. But there is an small increment in FMI score comparatively FMI score of clustering with $k$ value less than or equal to 20. Therefore, we have generalized the k-value as less than or equal to 20 . The sensitivity of parameter $k$ needs to be investigated more in future. The number of clusters is defined as a constant that is equal to the number of ground truth classes. The original class labels are not used anywhere in the experimental evaluation. The ground truth class labels are used only for measuring the FMI score that determines the cluster purity. The threshold parameter is set to 0.05 that provides the intuition for selecting the recommended clustering techniques from the order sequence. The threshold parameter derived from the statistical method where the maximum allowable difference between two consecutive values of either increasing or decreasing order sequence is $5 \%$. The recommended set size and threshold parameters are inversly proposional to each other. The sensitivity of threshold parameter needs to be investigated further. From the recommended set, the best technique is chosen and the optimal sample of it being considered as the best representative sample for designing the t-SNE model. The representative sample is embedded in a 2D space using the BH-tSNE algorithm. The parameters of BH-tSNE are set up according to the paper [13] experimental setup. In addition to that, we initialized the embedding space $\mathcal{Y}$ by sampling the point $y_{i}$ from a uniform distribution with $[-0.02,0.02]^{2}$ for obtaining well-separated clusters in embedding space. The datasets with more than 50 dimensions, their dimensionality is reduced to 50 by kernel PCA. The dimensionality reduction speeds up the computation of the probability distribution of the input similarity and suppresses some noise. The results of the BH-tSNE algorithm are shown in 2D scatter-plot representation. The minimum value of $p_{i j}$ of clustered data, the early exaggeration factor $\alpha$ and the learning rate $\eta$ values are assigned similar to the George C.linderman and Stefan Steinerberger paper.

The data points other than the representative samples are interpolated into the BH-tSNE of a representative sample using LIDWI. The parameter $r_{x}$ is obtained by either proposed heuristic or intuition of LION-tSNE. In the proposed heuristic dilation factor is bounded between 0 and 1 . The parameter $r_{y}, r_{y c l o s e}$ and power are measured similar to LION-tSNE algorithm.

Table 2: Parameters setting for the experimental setup

\begin{tabular}{ll}
\hline Parameter & Value \\
\hline k of $k$-NN sampling & $1 \leq k \leq 20$ \\
Threshold & 0.05 \\
Perplexity & $5-50$ \\
Early exaggeration coefficient & $\sim \frac{N}{10}$ \\
Adaptive learning rate & $1-200$ \\
Dilation Factor & $0 \leq d f \leq 1$ \\
$r_{x}$ at dist_perc & $95-100$ \\
$r_{y}$ at dist_perc & 100 \\
$r_{\text {yclose }}$ at dist_perc & 10 \\
Fixed k-value for k-NN accuracy & $3-10$ \\
\hline
\end{tabular}

Table 2 provides the parameter settings of our experimental evaluation. The parameter perplexity represents the effective number of neighbors for each data point. For instance, the small value of perplexity creates subgroups within the same cluster of t-SNE results. In contrast to small, the large value of it does not maintain the clear separation between two clusters of t-SNE results. Both cases suffer from either under-fitting or over-fitting problem that causes a lack of visual clarity. The empirical studies state that the perplexity value between 5 to 70 gives a good visual representation of t-SNE results. The parameter dist per represents the overall percentile of the representative sample that needs to be considered as inliers. For 


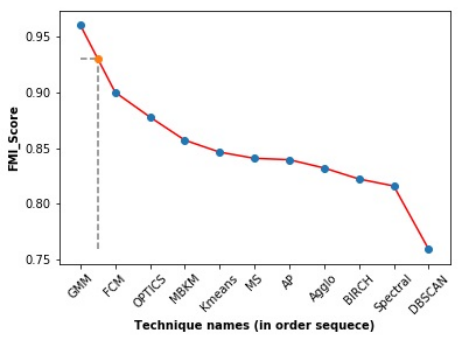

(a) IRIS

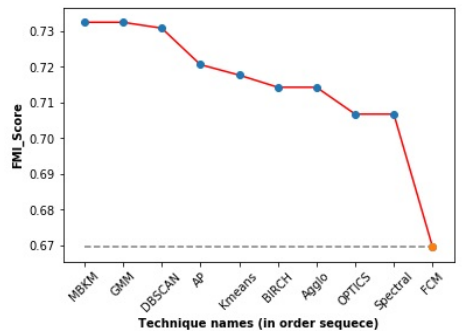

(e) Leukemia-ALL-AML

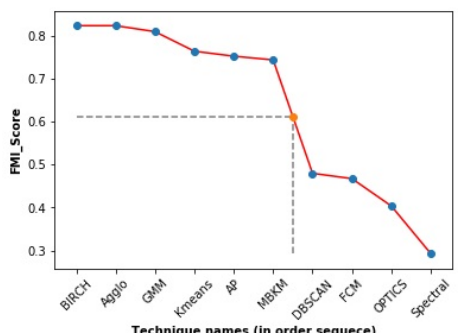

(i) MNIST

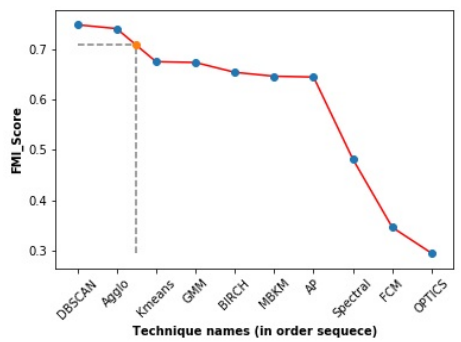

(m) COIL-20

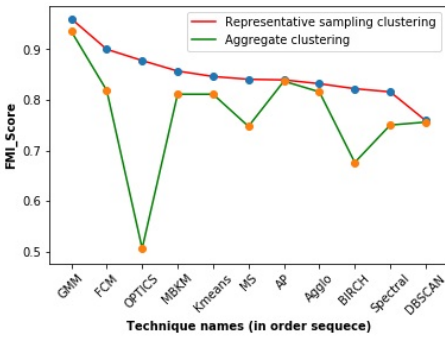

(b) IRIS

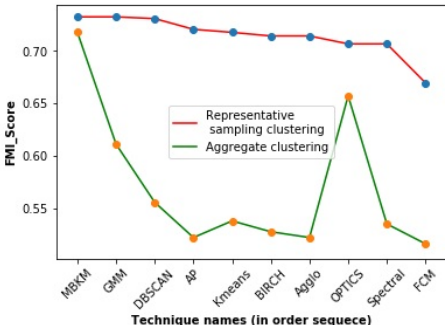

(f) Leukemia-ALL-AML

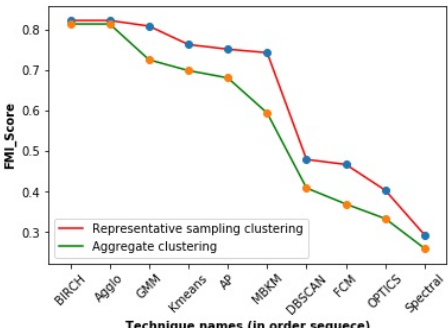

(j) MNIST

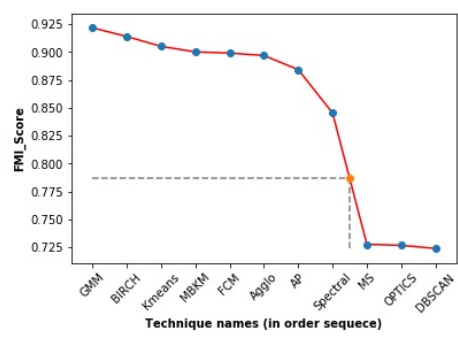

(c) Breast-Cancer

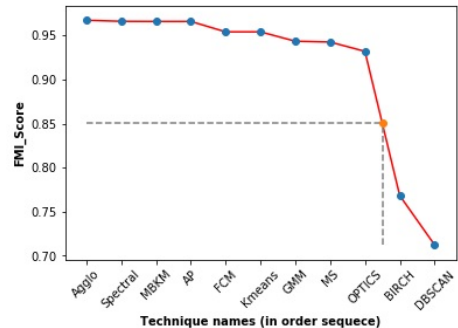

(g) Wine

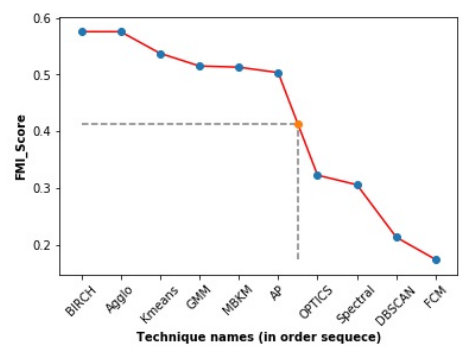

(k) Olivetti-Faces

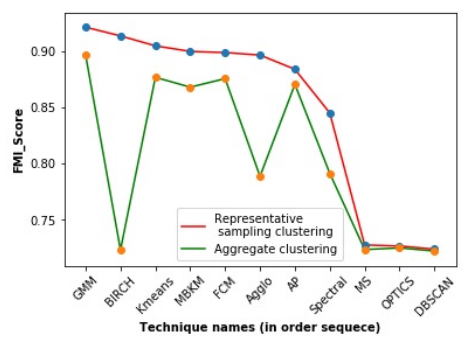

(d) Breast-Cancer

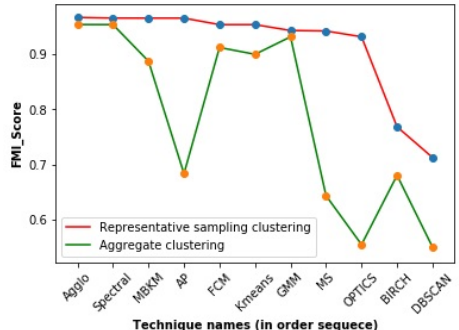

(h) Wine

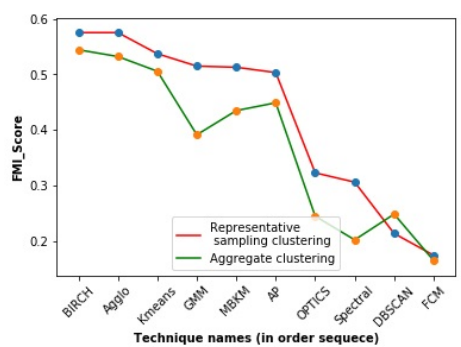

(1) Olivetti-Faces

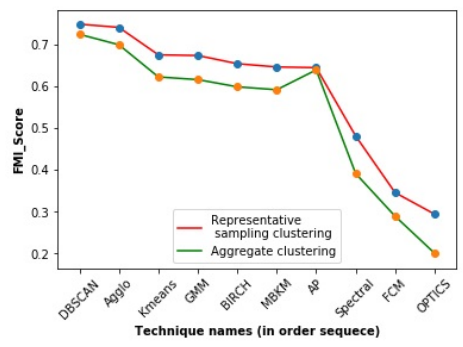

(n) COIL-20

Figure 4: The column one and column three (i.e., sub-figure $((a),(c),(e),(g),(i),(k)$ and $(m))$ shows the threshold derivation (i.e., the integration of doted horizontal and vertical lines) of all seven datasets from the order sequence curve. The column two and column four (i.e., sub-figure $(b),(d),(f),(h),(j),(l)$ and $(n))$ shows the relationship curve of all datasets from the order sequence, which provides the relationship between the FMI scores of proposed representative $k$-NN sampling-based clustering and aggregate clustering.

example, if we take dist_per as 95th percentile, that means out of 100 points, 95 points are considered as inliers and the remaining 5 points are outliers. The parameter $k$ plays an important role in the computation of $k$-NN accuracy of the data. The effect of parameter $k$ is shown in the Figure 3 It is clear that when there is an increment in $k$ value, accuracy decreases monotonically. The result evaluation of representative $k$-NN sampling-based clustering is discussed in the next section.

\subsection{Result analysis}

In our experimentation, we have considered most frequently and popularly used clustering techniques. The Figure 4 shows the curves of all seven datasets of subsection 5.1. The Figure 4 gives the threshold derivation for the recommended order sequence and relationship between the FMI scores of proposed representative $k$-NN sampling-based clustering and aggregate clustering. In the Figure 4. the $\mathrm{x}$-axis represent the clustering technique names in the order sequence of FMI_scores (i.e., $F M I\left(t_{1}\right)>F M I\left(t_{2}\right)>\ldots .>F M I\left(t_{l}\right)$ where $t_{i}$ represents $i^{\text {th }}$ best clustering technique) and y-axis represents the corresponding FMI_Scores. Column one and column three of Figure 4 (i.e., sub-figure $(a),(c),(e),(g),(i),(k)$ and $(m))$ shows the threshold derivation for the recommendation of clustering techniques for all the given seven datasets. The column two and column four of Figure 4 (i.e., sub-figure $(b),(d),(f),(h),(j),(l)$ and $(n))$ 
Table 3: The applicable clustering and their recommended order sequence for the given seven datasets.

\begin{tabular}{|c|c|c|}
\hline Dataset & Applicable cluster methods & Recommended order sequence of cluster methods \\
\hline IRIS & $\begin{array}{l}\text { k-Means, Agglomerative, BIRCH, Spectral, EMGMM, FCM, DBSCAN, AP, } \\
\text { MBKM, OPTICS, MS }\end{array}$ & EMGMM \\
\hline Breast-cancer & $\begin{array}{l}\text { k-Means, Agglomerative, BIRCH, Spectral, EMGMM, FCM, DBSCAN, AP, } \\
\text { MBKM, OPTICS, MS }\end{array}$ & $\begin{array}{l}\text { EMGMM, BIRCH, k-Means, MBKM, FCM, Agglomerative, AP, Spec- } \\
\text { tral }\end{array}$ \\
\hline Leukemia & $\begin{array}{l}\text { k-Means, Agglomerative, BIRCH, Spectral, EMGMM, FCM, DBSCAN, AP, } \\
\text { MBKM, OPTICS }\end{array}$ & $\begin{array}{l}\text { MBKM, EMGMM, DBSCAN, AP, k-Means, BIRCH, Agglomerative, } \\
\text { OPTICS, Spectral, FCM }\end{array}$ \\
\hline Wine & $\begin{array}{l}\text { k-Means, Agglomerative, BIRCH, Spectral, EMGMM, FCM, DBSCAN, AP, } \\
\text { MBKM, OPTICS, MS }\end{array}$ & $\begin{array}{l}\text { Agglomerative, Spectral, MBKM, AP, FCM, k-Means, EMGMM, MS, } \\
\text { OPTICS }\end{array}$ \\
\hline MNIST & $\begin{array}{l}\text { k-Means, Agglomerative, BIRCH, Spectral, EMGMM, FCM, DBSCAN, AP, } \\
\text { MBKM, OPTICS }\end{array}$ & BIRCH, Agglomerative, EMGMM, k-Means, AP, MBKM \\
\hline Olivetti-faces & $\begin{array}{l}\text { k-Means, Agglomerative, BIRCH, Spectral, EMGMM, FCM, DBSCAN, AP, } \\
\text { MBKM, OPTICS }\end{array}$ & BIRCH, Agglomerative, k-Means, EMGMM, MBKM, AP \\
\hline COIL-20 & $\begin{array}{l}\text { k-Means, Agglomerative, BIRCH, Spectral, EMGMM, FCM, DBSCAN, AP, } \\
\text { MBKM, OPTICS }\end{array}$ & DBSCAN, Agglomerative \\
\hline
\end{tabular}

shows the relationship curve that provides the relationship between the FMI_Score of representative $k$-NN sampling-based clustering and aggregate clustering for all the given seven datasets. The Figure 4 clearly shows that the proposed representative $k$-NN samplingbased clustering results are superior to the aggregate clustering results.

The sets of applicable and recommended order sequence of clustering techniques for the given seven datasets is listed in the Table 3 The $\mathrm{CP}$ and $\mathrm{HCI}$ of representative $k$-NN sampling-based clustering and aggregate clustering of all clustering techniques are listed in the Table 4 and 5 . The Table 4 and 5 clearly states that the $\mathrm{CP}$ of the proposed representative $k$-NN sampling-based clustering is smaller than the aggregate clustering for some techniques. In contrary techniques, the compactness is in reverse order, but the compactness difference is very minute in both situations. The CHI is also deriving the relationship between both clustering as same as $\mathrm{CP}$ where the $\mathrm{CP}$ is small, and then there is a large $\mathrm{CHI}$ score.

The contingency matrices of representative $k$-NN samplingbased clustering and aggregate clustering of IRIS, Wine, Breastcancer and Leukemia datasets are shown in the Table 6, $7,8,8$ and 9 If the number of classes of the given dataset is larger, then it is difficult to analyze the data with the contingency matrix. Therefore, the contingency matrix of MNIST, Olivetti-Faces and COIL-20 datasets are not addressed. The Table 6, 7, 8 and 9 clearly states that the proposed representative $k$-NN sampling-based clustering classifies the labels much similar to the ground truth class labels compared to aggregate clustering. The Tabel 9 represents the contingency matrix of the Leukemia dataset, which indicates that none of the selected technique gives the best clustering results. Still, representative $k$-NN sampling-based clustering produces better results than the aggregate clustering.

For Quantitative evaluation, the baseline $k$-NN accuracy of proposed representative $k$-NN sampling-based clustering is compared with baseline $k$-NN accuracy of aggregate clustering and baseline $k$-NN accuracy of ground truth class labels. The baseline $k$-NN accuracy and trustworthiness of representative $k$-NN sampling-based clustering, aggregate clustering and ground truth class labels of seven datasets are listed in Table 10 . The Table 10 clearly indicates that the proposed method produces more robust results than others.
Table 4: Compactness and CHI score of representative $k$-NN sampling-based clustering, overall sampling clustering (i.e., assigning the cluster labels based on the KNN of remaining samples in the context of representative samples) and the aggregate clustering of IRIS, Breast-Cancer, Leukemia and Wine datasets for the selected clustering techniques.

\begin{tabular}{|c|c|c|c|c|c|c|c|}
\hline \multirow[t]{2}{*}{ Method } & \multirow{2}{*}{$\begin{array}{l}\text { Samp. } \\
\text { size }\end{array}$} & \multicolumn{3}{|c|}{ Compactness } & \multicolumn{3}{|c|}{ CHI Score } \\
\hline & & $\begin{array}{l}\text { Samp. } \\
\text { cltr }\end{array}$ & $\begin{array}{l}\text { Overall } \\
\text { cltr }\end{array}$ & $\begin{array}{l}\text { Aggr. } \\
\text { cltr }\end{array}$ & $\begin{array}{l}\text { Samp. } \\
\text { cltr }\end{array}$ & $\begin{array}{l}\text { Overall } \\
\text { cltr }\end{array}$ & $\begin{array}{l}\text { Aggr. } \\
\text { cltr }\end{array}$ \\
\hline$k$-Means & 73 & 0.4945 & 0.5601 & 0.5557 & 221.54 & 347.56 & 358.56 \\
\hline Agglomerative & 63 & 0.5143 & 0.5579 & 0.5606 & 178.78 & 353.21 & 348.03 \\
\hline Birch & 73 & 0.5291 & 0.5646 & 0.8507 & 183.85 & 335.22 & 193.97 \\
\hline Spectral & 60 & 0.5234 & 0.5606 & 0.5709 & 167.457 & 348.03 & 322.48 \\
\hline EMGMM & 63 & 0.5316 & 0.5754 & 0.5789 & 161.44 & 317.59 & 307.76 \\
\hline FCM & 72 & 0.5107 & 0.5633 & 0.5576 & 197.71 & 340.79 & 355.71 \\
\hline DBSCAN & 73 & 0.6379 & 0.7047 & 0.6723 & 104.84 & 175.96 & 124.78 \\
\hline $\mathrm{AP}$ & 73 & 0.52 & 0.557 & 0.5588 & 196.99 & 354.54 & 353.22 \\
\hline MBKM & 74 & 0.4834 & 0.5613 & 0.5562 & 234.32 & 343.95 & 356.28 \\
\hline OPTICS & 73 & 0.5894 & 0.6071 & 1.0919 & 120.53 & 259.45 & 15.99 \\
\hline MS & 70 & 0.527 & 0.557 & 0.6002 & 188.2 & 354.45 & 289.52 \\
\hline \multicolumn{8}{|l|}{ Breast-Cancer } \\
\hline$k$-Means & 291 & 1.5608 & 1.6064 & 1.6013 & 203.91 & 355.42 & 364.09 \\
\hline Agglomerative & 290 & 1.5735 & 1.6073 & 1.6322 & 199.73 & 356.80 & 319.01 \\
\hline Birch & 291 & 1.5698 & 1.6083 & 1.9509 & 193.53 & 352.67 & 53.96 \\
\hline Spectral & 289 & 1.5694 & 1.6177 & 1.6449 & 197.34 & 351.45 & 328.74 \\
\hline EMGMM & 276 & 1.5744 & 1.6096 & 1.6168 & 178.38 & 350.08 & 337.00 \\
\hline FCM & 276 & 1.5645 & 1.6048 & 1.6001 & 186.30 & 358.22 & 363.04 \\
\hline DBSCAN & 291 & 1.9493 & 2.0004 & 1.9735 & 21.27 & 19.66 & 38.11 \\
\hline $\mathrm{AP}$ & 213 & 1.5146 & 1.6088 & 1.6088 & 156.95 & 356.82 & 358.62 \\
\hline MBKM & 268 & 0.8824 & 1.6047 & 1.6007 & 180.883 & 359.63 & 363.83 \\
\hline OPTICS & 268 & 1.9635 & 2.0179 & 2.0069 & 21.72 & 24.65 & 38.41 \\
\hline MS & 213 & 1.9138 & 2.0054 & 1.9412 & 19.79 & 17.65 & 68.97 \\
\hline \multicolumn{8}{|c|}{ Leukemia-ALL-AML } \\
\hline$k$-Means & 22 & 3.4413 & 3.9367 & 3.9279 & 1.567 & 1.173 & 1.685 \\
\hline Agglomerative & 19 & 3.3494 & 3.9315 & 3.9222 & 1.874 & 1.269 & 1.756 \\
\hline Birch & 19 & 3.3494 & 3.9315 & 3.919 & 1.874 & 1.269 & 1.921 \\
\hline Spectral & 19 & 3.3076 & 3.915 & 3.92 & 1.707 & 1.279 & 1.845 \\
\hline EMGMM & 20 & 3.4012 & 3.9415 & 3.9237 & 1.337 & 0.953 & 1.591 \\
\hline FCM & 20 & 3.3743 & 3.939 & 3.9271 & 1.622 & 1.117 & 1.619 \\
\hline DBSCAN & 17 & 3.2654 & 3.9436 & 3.9225 & 1.351 & 0.959 & 1.326 \\
\hline $\mathrm{AP}$ & 14 & 2.8993 & 3.895 & 3.9355 & 1.548 & 0.882 & 1.292 \\
\hline MBKM & 22 & 3.3088 & 3.8957 & 3.9366 & 1.102 & 0.859 & 1.122 \\
\hline OPTICS & 19 & 3.315 & 3.922 & 3.9071 & 0.859 & 0.717 & 1.317 \\
\hline MS & $\ldots$ & $\ldots$ & $\ldots$. & $\ldots$. & $\ldots$. & $\ldots$. & $\ldots$. \\
\hline \multicolumn{8}{|l|}{ Wine } \\
\hline$k$-Means & 82 & 1.3275 & 1.4298 & 1.4289 & 47.255 & 82.828 & 83.373 \\
\hline Agglomerative & 87 & 1.3207 & 1.4356 & 1.4328 & 45.964 & 80.465 & 81.327 \\
\hline Birch & 82 & 1.555 & 1.6567 & 1.629 & 19.859 & 34.395 & 42.564 \\
\hline Spectral & 87 & 1.3532 & 1.4348 & 1.4298 & 42.372 & 81.014 & 82.828 \\
\hline EMGMM & 71 & 1.3481 & 1.4331 & 1.4348 & 39.846 & 81.796 & 81.698 \\
\hline FCM & 85 & 1.3473 & 1.4317 & 1.4305 & 45.029 & 82.346 & 83.135 \\
\hline DBSCAN & 64 & 1.6181 & 1.6656 & 1.9606 & 15.719 & 37.502 & 3.102 \\
\hline $\mathrm{AP}$ & 82 & 1.5534 & 1.3368 & 1.4458 & 45.362 & 80.714 & 80.828 \\
\hline MBKM & 87 & 1.3562 & 1.4848 & 1.4278 & 42.472 & 81.784 & 81.523 \\
\hline OPTICS & 85 & 1.4484 & 1.4531 & 1.4248 & 38.866 & 82.796 & 81.698 \\
\hline MS & 71 & 1.3489 & 1.4371 & 1.4748 & 49.846 & 81.797 & 80.598 \\
\hline
\end{tabular}


Table 5: Compactness and CHI score of representative $k$-NN sampling-based clustering, overall sampling clustering (i.e., assigning the cluster labels based on the KNN of remaining samples in the context of representative samples) and the aggregate clustering of MNIST Handwritten digits, Olivetti-Faces and COIL-20 datasets for the selected clustering techniques.

\begin{tabular}{|c|c|c|c|c|c|c|c|}
\hline \multirow[t]{2}{*}{ Method } & \multirow{2}{*}{$\begin{array}{l}\text { Samp. } \\
\text { size }\end{array}$} & \multicolumn{3}{|c|}{ Compactness } & \multicolumn{3}{|c|}{ CHI Score } \\
\hline & & $\begin{array}{l}\text { Samp. } \\
\text { cltr }\end{array}$ & $\begin{array}{l}\text { Overall } \\
\text { cltr }\end{array}$ & $\begin{array}{l}\text { Aggr. } \\
\text { cltr }\end{array}$ & $\begin{array}{l}\text { Samp. } \\
\text { cltr }\end{array}$ & $\begin{array}{l}\text { Overall } \\
\text { cltr }\end{array}$ & $\begin{array}{l}\text { Aggr. } \\
\text { cltr }\end{array}$ \\
\hline$k$-Means & 4481 & 3816.4 & 3897.8 & 3877.8 & 233.97 & 477.45 & 492.48 \\
\hline Agglomerative & 4900 & 3936.2 & 3974.2 & 3974.2 & 206.16 & 409.22 & 395.43 \\
\hline Birch & 4900 & 3936.2 & 3974.2 & 3974.2 & 206.16 & 409.22 & 395.43 \\
\hline Spectral & 4735 & 4662.2 & 4694.5 & 4695.0 & 1.048 & 1.0308 & 1.0459 \\
\hline EMGMM & 4206 & 3862.2 & 3930.6 & 3977 & 200.8 & 443.59 & 400.32 \\
\hline FCM & 4835 & 3962.2 & 4374.5 & 4555. & 1.248 & 1.308 & 1.59 \\
\hline DBSCAN & 4735 & 4662.2 & 4694.5 & 4695. & 1.048 & 1.0308 & 1.0459 \\
\hline $\mathrm{AP}$ & 4496 & 3832.5 & 3978.6 & 3897.3 & 213.97 & 457.78 & 472.34 \\
\hline MBKM & 4783 & 4216.4 & 4597.1 & 4577.3 & 224.17 & 467.65 & 452.18 \\
\hline OPTICS & 4625 & 3962.5 & 4094.3 & 4195.7 & 1.258 & 1.13 & 1.045 \\
\hline MS & $\ldots$ & $\ldots$. & $\ldots$. & $\ldots$. & $\ldots$ & $\ldots$. & $\ldots$. \\
\hline \multicolumn{8}{|l|}{ Olivetti-Faces } \\
\hline$k$-Means & 200 & 1568 & 1784.5 & 1565.8 & 10.384 & 14.009 & 21.210 \\
\hline Agglomerative & 178 & 1495.9 & 1798.8 & 1546.8 & 10.718 & 13.634 & 22.008 \\
\hline Birch & 178 & 1495.9 & 1798.8 & 1546.8 & 10.718 & 13.634 & 22.008 \\
\hline Spectral & 188 & 2235 & 2545.9 & 2608.4 & 0.6858 & 0.6839 & 0.9924 \\
\hline EMGMM & 165 & 1528.9 & 1812.8 & 1591.5 & 9.211 & 13.168 & 20.272 \\
\hline FCM & 178 & 2479.3 & 2659.4 & 2732.6 & 4.321 & 4.874 & 4.178 \\
\hline DBSCAN & 194 & 2679.4 & 2750.5 & 2745.1 & 4.021 & 3.804 & 5.782 \\
\hline $\mathrm{AP}$ & 194 & 1628.9 & 1852.8 & 1691.5 & 10.321 & 14.168 & 20.872 \\
\hline MBKM & 178 & 1668.6 & 1852.8 & 1671.5 & 9.711 & 12.168 & 21.275 \\
\hline OPTICS & 165 & 1598.4 & 1932.8 & 1891.5 & 8.217 & 13.468 & 22.728 \\
\hline MS & $\ldots$ & $\ldots$. & $\ldots$ & $\ldots$ & $\ldots$. & $\ldots$. & $\ldots$ \\
\hline \multicolumn{8}{|l|}{ COIL-20 } \\
\hline$k$-Means & 679 & 10.333 & 10.362 & 10.180 & 87.911 & 183.571 & 188.454 \\
\hline Agglomerative & 701 & 10.239 & 10.237 & 10.294 & 90.220 & 184.703 & 181.461 \\
\hline Birch & 701 & 10.577 & 10.562 & 11.1165 & 87.377 & 180.283 & 162.538 \\
\hline Spectral & 676 & 14.852 & 14.944 & 17.447 & 28.585 & 57.964 & 27.4288 \\
\hline EMGMM & 679 & 10.273 & 10.243 & 10.356 & 87.832 & 186.73 & 187.686 \\
\hline FCM & 689 & 12.872 & 13.645 & 15.745 & 34.784 & 56.768 & 34.58 \\
\hline DBSCAN & 679 & 18.843 & 18.962 & 19.769 & 19.532 & 38.902 & 38.548 \\
\hline $\mathrm{AP}$ & 701 & 10.573 & 10.253 & 10.856 & 83.83 & 188.63 & 186.656 \\
\hline MBKM & 679 & 10.253 & 10.143 & 10.366 & 80.832 & 188.73 & 187.656 \\
\hline OPTICS & 679 & 10.243 & 10.233 & 10.326 & 87.432 & 185.738 & 184.286 \\
\hline MS & $\ldots$ & $\ldots$ & $\ldots$ & $\ldots$ & $\ldots$ & $\ldots$ & $\ldots$ \\
\hline
\end{tabular}

Table 6: Contingency matrix of $k$-NN sampling-based and original clustering on IRIS dataset with best $k$-NN sampling-based clustering technique (i.e., EMGMM).

\begin{tabular}{|c|c|c|c|c|c|c|}
\hline & \multicolumn{6}{|c|}{ Class labels generated by } \\
\hline & \multicolumn{3}{|c|}{$\begin{array}{l}k \text {-NN sampling- } \\
\text { based clustering }\end{array}$} & \multicolumn{3}{|c|}{ Aggregate clustering } \\
\hline & C1 & C2 & $\mathrm{C} 3$ & C1 & $\mathrm{C} 2$ & $\mathrm{C3}$ \\
\hline \multirow{3}{*}{ 压 } & 50 & 0 & 0 & 50 & 0 & 0 \\
\hline & 0 & 49 & 1 & 0 & 45 & 5 \\
\hline & 0 & 2 & 48 & 0 & 0 & 50 \\
\hline
\end{tabular}

Table 7: Contingency matrix of $k$-NN sampling-based and original clustering on Wine dataset with best $k$-NN sampling-based clustering technique (i.e., Agglomerative).

\begin{tabular}{|c|c|c|c|c|c|c|}
\hline & \multicolumn{6}{|c|}{ Class labels generated by } \\
\hline & \multicolumn{3}{|c|}{$\begin{array}{l}k \text {-NN sampling- } \\
\text { based clustering }\end{array}$} & \multicolumn{3}{|c|}{ Aggregate clustering } \\
\hline & $\mathrm{C1}$ & $\mathrm{C} 2$ & $\mathrm{C3}$ & C1 & $\mathrm{C} 2$ & C3 \\
\hline C1 & 0 & 0 & 59 & 2 & 0 & 57 \\
\hline $\mathrm{C} 2$ & 69 & 2 & 0 & 69 & 2 & 0 \\
\hline C3 & 1 & 47 & 0 & 0 & 48 & 0 \\
\hline
\end{tabular}

The sampling $k$-NN accuracies such as train, test and overall of $\mathrm{t}$-SNE embedding of a representative sample and interpolation of other samples are listed in Table 11 and the overall trustworthiness is also covered. The best representative sample of representative $k$-NN sampling-based clustering and random sampling of IRIS dataset is shown in Figure 5. The 2D scatter-plots shown in Figure 5 are the first two coordinates of IRIS dataset. The Figure $5(a)$ shows the best representative sample of $k$-NN sampling and the Figure $5(c)$ shows the representative sample of $k$-NN sampling along with samples of $(\mathrm{t}, \mathrm{m}, \mathrm{s})-\mathrm{Nets}$ of remaining data points. The Figure $5 \mathrm{~b} b)$ and $5(d)$ shows the random sampling of size equal to the sample size of Figure 5 $(a)$ and $5(c)$. The samples of Figure 5(a) are constant and consistent comparatively other sampling.

Table 8: Contingency matrix of $k$-NN sampling-based and original clustering on Breast-Cancer dataset with best $k$-NN sampling-based clustering technique (i.e., EMGMM).

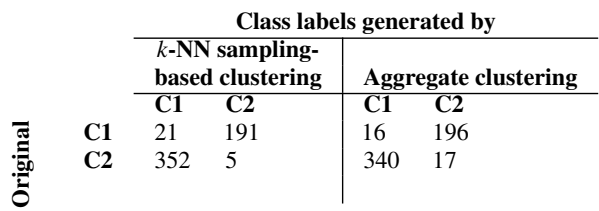

Table 9: Contingency matrix of $k$-NN sampling-based and original clustering on Leukemia dataset with best $k$-NN sampling-based clustering technique (i.e., MBKM)

\begin{tabular}{|c|c|c|}
\hline & \multicolumn{2}{|c|}{ Class labels generated by } \\
\hline & $\begin{array}{l}k \text {-NN sampling- } \\
\text { based clustering }\end{array}$ & Aggregate clustering \\
\hline & C1 C2 & $\mathrm{C1} \mathrm{C2}$ \\
\hline C1 & 47 & 38 \\
\hline 言 & 23 & 19 \\
\hline
\end{tabular}

Table 10: Baseline $k$-NN accuracy of $k$-NN sampling based clustering, original clustering and ground truth class labels

\begin{tabular}{lllll}
\hline \multirow{2}{*}{$\begin{array}{l}\text { Dataset } \\
\text { Name }\end{array}$} & Trust & \multicolumn{3}{c}{ Baseline $k$-NN Accuracy } \\
\cline { 3 - 5 } & & $\begin{array}{l}\text { Sampling } \\
\text { Clustering }\end{array}$ & $\begin{array}{l}\text { Aggregate } \\
\text { Clustering }\end{array}$ & $\begin{array}{l}\text { Ground-truth } \\
\text { class labels }\end{array}$ \\
\hline IRIS & 0.9861 & 0.9735 & 0.9666 & 0.96 \\
Breast-Cancer & 0.958 & 0.9876 & 0.9862 & 0.9577 \\
Leukemia & 0.6799 & 0.9875 & 0.6643 & 0.7485 \\
Wine & 0.9552 & 0.9774 & 0.9828 & 0.9717 \\
MNIST Digits & 0.9895 & 0.9259 & 0.9483 & 0.9512 \\
Olivetti face & 0.9494 & 0.6775 & 0.8287 & 0.88 \\
COIL-20 & 0.9972 & 0.9554 & 0.9709 & 0.9743 \\
\hline
\end{tabular}

The Figure 6 shows the 2D scatter-plot visualization of IRIS data with outliers projection. In Figure 6, we are also showing the outliers (i.e. denoted by large grey color circle) of original data after finding the outliers from the addition of remaining data points with the radius $r_{x}$ which is obtained by the proposed heuristic $r_{x}=(1+d f) * r_{x}$ with $d f=0.2$. It clearly states that sampled data obtain the outliers of original data.

The 2D scatter-plot visualization of embedding space of representative $k$-NN sampling-based clustering of the MNIST dataset is shown in the Figure 7. The Figure 7 shows the baseline 2D scatter-plot of t-SNE embedding of whole MNIST data of size 10K 
Table 11: Quantitative measure using $k$-NN accuracy of $k$-NN sampling based clustering, original clustering and ground truth class labels

\begin{tabular}{|c|c|c|c|c|c|c|c|c|c|c|c|}
\hline \multirow{3}{*}{$\begin{array}{l}\text { Dataset } \\
\text { Name }\end{array}$} & \multirow{3}{*}{$\begin{array}{l}\text { Sample } \\
\text { Size }\end{array}$} & \multirow[t]{3}{*}{ Trust } & \multicolumn{9}{|c|}{ Sampling $k$-NN Accuracy } \\
\hline & & & \multicolumn{3}{|c|}{ Sampling-based clustering } & \multicolumn{3}{|c|}{ aggregate clustering } & \multicolumn{3}{|c|}{ Ground truth class labels } \\
\hline & & & Train & Test & Overall & Train & Test & Overall & Train & Test & Overall \\
\hline IRIS & 73 & 0.9594 & 0.9726 & 0.9594 & 0.9661 & 0.9753 & 0.9405 & 0.9652 & 0.9589 & 0.9324 & 0.9585 \\
\hline Breast Cancer & 291 & 0.9239 & 0.9896 & 0.9856 & 0.9820 & 0.9690 & 0.9856 & 0.9876 & 0.9452 & 0.9676 & 0.9546 \\
\hline Leukemia & 33 & 0.5665 & 0.9416 & 1.0 & 0.9875 & 0.6714 & 0.4102 & 0.6266 & 0.6363 & 0.5897 & 0.6247 \\
\hline Wine & 87 & 0.9299 & 0.9377 & 0.956 & 0.9379 & 0.966 & 0.967 & 0.9604 & 0.9511 & 0.945 & 0.9548 \\
\hline MNIST & 4900 & 0.9635 & 0.9346 & 0.8956 & 0.9120 & 0.9201 & 0.91 & 0.9206 & 0.9335 & 0.8972 & 0.9170 \\
\hline Olivetti-Faces & 200 & 0.968 & 0.6076 & 0.5128 & 0.6045 & 0.6356 & 0.7948 & 0.8270 & 0.6334 & 0.7794 & 0.8578 \\
\hline COIL-20 & 701 & 0.9941 & 0.9841 & 0.9417 & 0.9509 & 0.9529 & 0.9634 & 0.9749 & 0.9426 & 0.9688 & 0.9727 \\
\hline \multicolumn{12}{|c|}{ Random Sample of size equal to optimal sample } \\
\hline IRIS & 73 & 0.9654 & 0.9726 & 0.9859 & 0.98 & 0.9808 & 0.9802 & 0.977 & 0.9616 & 0.9661 & 0.9728 \\
\hline Breast Cancer & 291 & 0.9337 & 0.9759 & 0.9712 & 0.9753 & 0.9828 & 0.9892 & 0.9929 & 0.9553 & 0.9496 & 0.9472 \\
\hline Leukemia & 33 & 0.5495 & 1.0 & 0.9722 & 0.9866 & 5654 & 0.6111 & 0.6133 & 0.5771 & 0.4444 & 0.6087 \\
\hline Wine & 87 & 0.9316 & 0.9639 & 0.9662 & 0.9517 & 0.9431 & 0.9662 & 0.949 & 0.9671 & 0.9775 & 0.9547 \\
\hline MNIST & 4900 & 0.9599 & 0.9122 & 0.8918 & 0.9001 & 0.9157 & 0.8977 & 0.9081 & 0.9249 & 0.8956 & 0.9048 \\
\hline Olivetti-Faces & 200 & 0.9578 & 0.5080 & 0.5050 & 0.5860 & 0.7235 & 0.7121 & 0.7869 & 0.6967 & 0.6767 & 0.7842 \\
\hline COIL-20 & 701 & 0.9935 & 0.9543 & 0.9362 & 0.9449 & 0.9699 & 0.9606 & 0.9652 & 0.9273 & 0.9375 & 0.9515 \\
\hline \multicolumn{12}{|c|}{ Optimal Sample plus Lhs Sample } \\
\hline IRIS & 79 & 0.965 & 0.9746 & 1.0 & 0.9797 & 0.9733 & 0.9852 & 0.9862 & 0.9594 & 0.9852 & 0.9632 \\
\hline Breast Cancer & 305 & 0.9348 & 0.9736 & 0.9772 & 0.9806 & 0.9934 & 0.9848 & 0.9912 & 0.9608 & 0.9810 & 0.9701 \\
\hline Leukemia & 41 & 0.5979 & 0.9777 & 1.0 & 0.9723 & 0.5361 & 0.6551 & 0.5994 & 0.5964 & 0.6551 & 0.6285 \\
\hline Wine & 95 & 0.9356 & 0.956 & 0.9512 & 0.9488 & 0.9461 & 0.9634 & 0.9717 & 0.9671 & 0.9512 & 0.9545 \\
\hline MNIST & 5327 & 0.9684 & 0.9289 & 0.9092 & 0.9144 & 0.9195 & 0.9158 & 0.9210 & 0.9276 & 0.9073 & 0.9180 \\
\hline Olivetti-Faces & 211 & 0.9641 & 0.6591 & 0.5271 & 0.6175 & 0.6418 & 0.7826 & 0.8492 & 0.6888 & 0.7989 & 0.886 \\
\hline COIL-20 & 729 & 0.9942 & 0.9705 & 0.9464 & 0.9536 & 0.9425 & 0.9690 & 0.9743 & 0.9263 & 0.9760 & 0.9785 \\
\hline \multicolumn{12}{|c|}{ Random Sample of size equal to Optimal Sample plus (t,m,s)-Nets Sample } \\
\hline IRIS & 79 & 0.9626 & 1.0 & 0.9705 & 0.9661 & 0.9772 & 0.9558 & 0.9657 & 0.9866 & 0.9705 & 0.9728 \\
\hline Breast Cancer & 305 & 0.9339 & 0.9802 & 0.9734 & 0.9806 & 0.9902 & 0.9924 & 0.9894 & 0.9542 & 0.9772 & 0.9683 \\
\hline Leukemia & 41 & 0.5557 & 0.9492 & 1.0 & 0.9732 & 0.6333 & 0.6451 & 0.6365 & 0.7289 & 0.4516 & 0.679 \\
\hline Wine & 95 & 0.9434 & 0.9444 & 0.9518 & 0.949 & 0.9456 & 0.9638 & 0.9493 & 0.9666 & 0.9518 & 0.9487 \\
\hline MNIST & 5327 & 0.9616 & 0.9326 & 0.8977 & 0.9138 & 0.9316 & 0.8977 & 0.9172 & 0.9433 & 0.8959 & 0.9177 \\
\hline Olivetti-Faces & 211 & 0.9708 & 0.6642 & 0.4867 & 0.6011 & 0.6938 & 0.7671 & 0.8108 & 0.6673 & 0.7724 & 0.85 \\
\hline COIL-20 & 729 & 0.9949 & 0.9838 & 0.9381 & 0.9558 & 0.9381 & 0.9620 & 0.9721 & 0.9330 & 9648 & 0.9666 \\
\hline
\end{tabular}

with representative $k$-NN sampling-based clustering labels as the colors of scatter point groups.
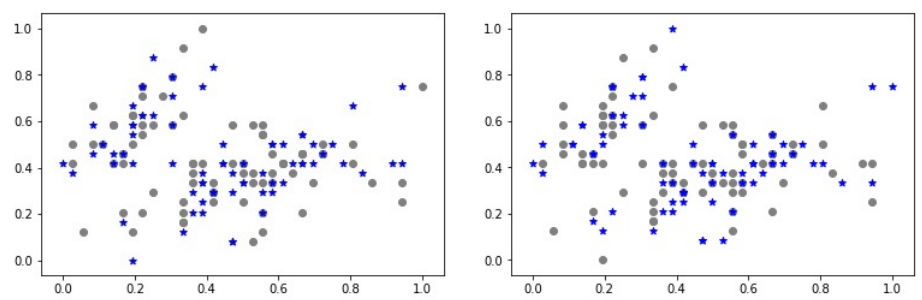

(a) Proposed representative $k$-NN sampling

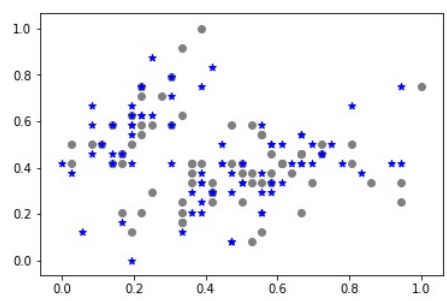

(b) Random sample of size equal to the size of proposed representative $k$-NN sampling

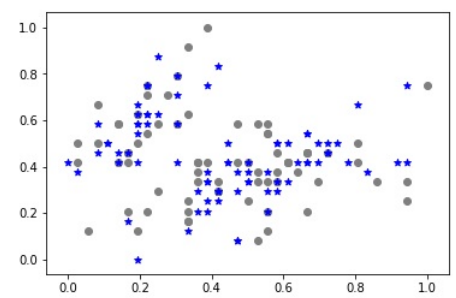

(c) Proposed representative $k$-NN sampling (d) Random sample of size equal to the size of along with the samples from remain samples proposed representative $k$-NN sampling plus using $(\mathrm{t}, \mathrm{m}, \mathrm{s})$-Nets $(\mathrm{t}, \mathrm{m}, \mathrm{s})$-Nets samples

Figure 5: Training sample selection (i.e., represented by blue star scatter-plot point) from IRIS dataset with four different strategies.

The Figure 8 shows the four different 2D scatter-plot representation of MNIST dataset. In Figure $8(a)$, we are representing the
2D scatter-plot of sampled t-SNE along with the interpolation of inliers and placement of outliers. The sampled t-SNE is designed based on the best representative sample of the representative $k$-NN sampling-based clustering concerning the best clustering technique. The inliers of remaining samples (i.e, other than representative samples) are interpolated to t-SNE with the parameter $r_{x}$ that is obtained from the proposed heuristic. The outlier of remaining samples are placed into an existing t-SNE environment based on the Boystov et.al heuristic.

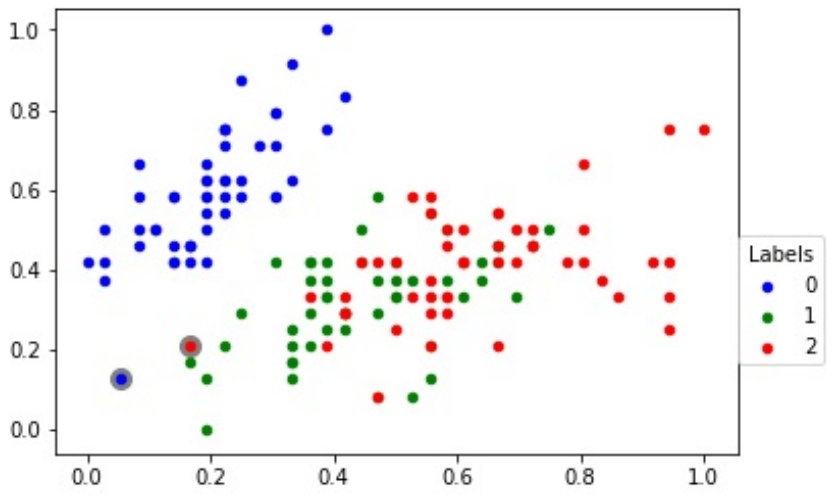

Figure 6: The 2D scatter-plot of IRIS data which denotes the outliers from the newly added sample with proposed heuristic.

In Figure $8(a)$, the outliers are placed separately from other embedding points. The Figure $8(b)$ shows the 2D scatter-plot of 
$(\mathrm{t}, \mathrm{m}, \mathrm{s})$-Nets based t-SNE along with interpolation of other samples. The $(\mathrm{t}, \mathrm{m}, \mathrm{s})$-Nets based $\mathrm{t}$-SNE is designed with a combination of representative $k$-NN sampling-based clustering and $(\mathrm{t}, \mathrm{m}, \mathrm{s})$-Nets of remaining samples. The $(\mathrm{t}, \mathrm{m}, \mathrm{s})$-Nets samples are selected from the data samples, which are having $\mathrm{NN}$-score as zero after selecting the samples using a mutual $k$-NN sampling with a static graph updation algorithm. The inliers of remaining samples (i.e, other than representative samples plus ( $\mathrm{t}, \mathrm{m}, \mathrm{s})$-Nets samples) are interpolated to t-SNE with the parameter $r_{x}$ that is obtained from the Boystov et.al heuristic. The Figure $8(c)$ and $8 d$ ) shows the 2D scatter-plots of a random sampled t-SNE along with the addition of new data samples. The random sample size is equal to the sample size of Figure $8(a)$ and $8(b)$. The inliers and outliers of remaining samples are placed according to the intuitions of Figure $8(a)$ and $8(b)$. The representative $k$-NN sampling-based results are statistically significant than the random sampling based results which is derived in our earlier paper [19]. The following section describe the conclusion and future work.

\section{Conclusion and Future Work}

In this paper, we have proposed a representative $k$-NN samplingbased clustering approach, which generates cluster results on a sampling basis. The most frequently used clustering techniques are applied to obtain the sampling-based cluster results. Initially, we determine the applicable set of techniques for the given dataset From the applicable set, sampling-based clustering results of each technique are evaluated by an external cluster validation index called FMI-score. The applicable techniques are arranged in an orderly sequence of their FMI scores. Some threshold parameter derives the recommendation of clustering techniques for the given dataset. From the recommended set, the first technique is selected as the most desirable clustering for the given dataset.

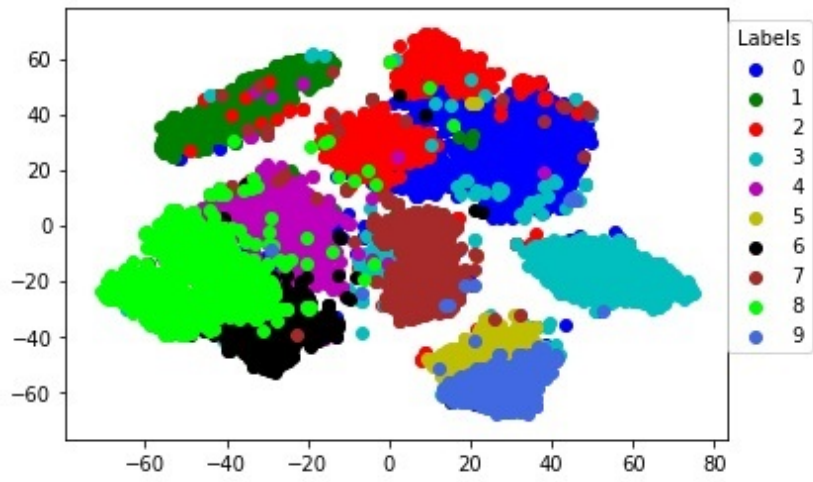

Figure 7: The baseline 2D visual representation of Representative $k$-NN samplingbased clustering t-SNE embedding where t-SNE applied on whole data

The samples of this technique are used for generating the lowdimensional embedding of input data. The embedding results are visualized and quantitatively evaluated in the context of samplingbased cluster results. The proposed approach is expanded to identify all the applicable set of clustering techniques for the given dataset, which can be done as future work. There is scope for implementing

the proposed algorithm in a distributed environment that can be applied to Big Data.

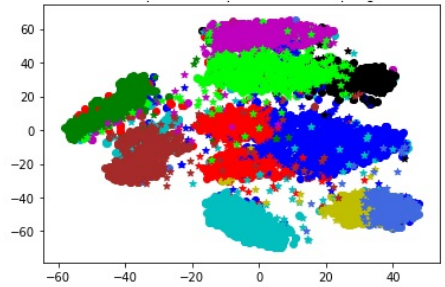

(a) Sampled t-SNE of representative sample

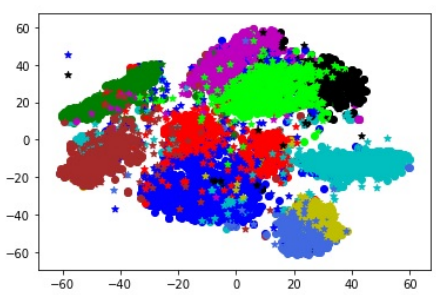

(c) Sampled t-SNE of random sample of size equal to representative sample size

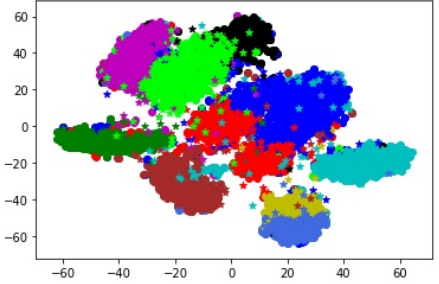

(b) Sampled t-SNE of representative sample plus $(\mathrm{t}, \mathrm{m}, \mathrm{s})$-Nets sample

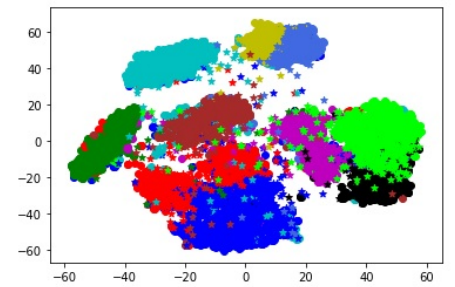

(d) Sampled t-SNE of random sample of size equal to representative sample plus $(\mathrm{t}, \mathrm{m}, \mathrm{s})$ Nets sample size

Figure 8: 2D representation of four different t-SNE embedding of MNIST dataset, (a) t-SNE embedding of representative train sample and the interpolation of inliers as well as placement of outliers with proposed heuristic, $(b) \mathrm{t}-\mathrm{SNE}$ embedding of representative train sample which includes the samples from $(\mathrm{t}, \mathrm{m}, \mathrm{s})$-Nets and the interpolation of inliers as well as placement of outliers with A Boystov et.al heuristic (c) t-SNE embedding of random sample of size equal to the size of sub-figure $(a)$ and the interpolation of inliers as well as placement of outliers with proposed heuristic, $(d)$ t-SNE embedding of random sample of size equal to the size of sub-figure $(b)$ and the interpolation of inliers as well as placement of outliers with A Boystov et.al heuristic.

Acknowledgment I would like to express my sincere gratitude to Visveswaraya $\mathrm{PhD}$ Scheme for providing financial support to pursue my PHD degree.

\section{References}

[1] M. Partridge, R.A. Calvo, "Fast dimensionality reduction and simple PCA" Intelligent data analysis, 2(3), 203-214, 1998.

[2] T.F. Cox, M.A. Cox, Multidimensional scaling, Chapman and hall/CRC, 2000.

[3] J. Shi, J. Malik, "Normalized cuts and image segmentation" IEEE Transactions on Pattern Analysis and Machine Intelligence, 22(8), 888-905, 2000, doi $10.1109 / 34.868688$

[4] J.B. Tenenbaum, V.D. Silva, J.C. Langford, "A global geometric framework for nonlinear dimensionality reduction” Science, 290(5500), 2319-2323, 2000. DOI: $10.1126 /$ science.290.5500.2319

[5] S.T. Roweis, L.K. Saul, "Nonlinear dimensionality reduction by locally linear embedding" Science, 290(5500), 2323-2326, 2000. DOI: 10.1126/science.290.5500.2323

[6] M. Belkin, P. Niyogi, "Laplacian Eigenmaps for dimensionality reduction and data representation" Neural computation, 15(6), 1373-1396, 2003. https://doi.org/10.1162/089976603321780317

[7] G.E. Hinton, S.T. Roweis, "Stochastic neighbor embedding" in Advances in neural information processing systems, pp. 857-864, 2003.

[8] L.V.D. Maaten, E. Postma, J.V.d. Herik, "Dimensionality Reduction: A Comparative" J Mach Learn Res, 10(13), 66-71, 2009.

[9] L.V.D. Maaten, G. Hinton, "Visualizing data using t-sne" J Mach Learn Res, 9 2579-2605, 2008. 
[10] W. Li, J.E. Cerise, Y. Yang, H. Han, "Application of t-sne to human genetic data" Journal of Bioinformatics and Computational Biology, 15(4) 1750017, 2017. https://doi.org/10.1142/S0219720017500172

[11] M. Nguyen, S. Purushotham, H. To, C. Shahabi, "m-tsne: A framework for visualizing high-dimensional multivariate time series" arXiv preprint arXiv:1708.07942, 2017 arXiv:1708.07942

[12] W.M. Abdelmoula, B. Balluff, S. Englert, J. Dijkstra, M.J.T. Reinders, A. Walch, L.A. McDonnell, B.P.F. Lelieveldt, "Data-driven identification of prognostic tumor subpopulations using spatially mapped t-sne of mass spectrometry imaging data" in Proceedings of the National Academy of Sciences, 113(43) 12244-12249, 2016. https://doi.org/10.1073/pnas.1510227113

[13] L.V.D. Maaten, "Accelerating t-sne using tree-based algorithms" J Mach Learn Res, 15, 3221-3245, 2014.

[14] N. Pezzotti, B.P.F. Lelieveldt, L.V.D. Maaten, T. Höllt, E. Eisemann, A. Vilanova, "Approximated and user steerable tsne for progressive visual analytics" IEEE transactions on visualization and computer graphics, 23(7) 1739-1752, 2016. DOI: $10.1109 /$ TVCG.2016.2570755

[15] L.V.D. Maaten, "Learning a parametric embedding by preserving local structure" in Artificial Intelligence and Statistics, pp 384-391, 2009.

[16] A. Gisbrecht, A. Schulz, B. Hammer, "Parametric nonlinear dimensionality reduction using kernel t-sne" Neurocomputing, 147, 71-82, 2015. https://doi.org/10.1016/j.neucom.2013.11.045

[17] A. Boytsov, F. Fouquet, T. Hartmann, Y. LeTraon, "Visualizing and exploring dynamic high-dimensional datasets with lion-tsne" arXiv preprint arXiv: 1708.04983, 2017. arXiv:1708.04983

[18] S. Ingram, T. Munzner, "Dimensionality reduction for documents with nearest neighbor queries" Neurocomputing, 150 557-569, 2015. https://doi.org/10.1016/j.neucom.2014.07.073

[19] B. Dharamsotu, K.S. Rani, S.A. Moiz, C.R. Rao, "k-NN sampling for visualization of dynamic data using LOIN-tSNE" in 2019 IEEE 26th International Conference on High Performance Computing, Data, and Analytics (HiPC), pages 63-72, 2019. DOI: 10.1109/HiPC.2019.00019

[20] L.J. Williams, H. Abdi, "Fishers least significant difference (1sd) test," Encyclopedia of research design, 218, 840-853, 2010.

[21] T. Kanungo, D.M. Mount, N.S. Netanyahu, C.D. Piatko, R. Silverman, A.Y. $\mathrm{Wu}$, "An efficient k-means clustering algorithm: Analysis and implementation" IEEE Transactions on Pattern Analysis and Machine Intelligence, 24(7) 881-892, 2002. DOI: 10.1109/TPAMI.2002.1017616

[22] W. Zhang, D. Zhao, X. Wang, "Agglomerative clustering via maximum incremental path integral" Pattern Recognition, 46(11) 3056-3065, 2013. https://doi.org/10.1016/j.patcog.2013.04.013

[23] T. Zhang, R. Ramakrishnan, M. Livny, "BIRCH: an efficient data clustering method for very large databases" ACM Sigmod Record, 25(2) 103-114, 1996. https://doi.org/10.1145/235968.233324

[24] $\mathrm{J}_{i}$ C. Bezdek, R. Ehrlich, W. Full, "FCM: The fuzzy c-means clustering algorithm" Computers and Geosciences, 10(2) 191-203, 1984.

[25] E. Schubert, J. Sander, M. Ester, H.P. Kriegel, X. Xu, "DBSCAN revisited, revisited: why and how you should (still) use DBSCAN" ACM Transactions on Database Systems (TODS), 42(3) 1-21, 2017. https://doi.org/10.1145/3068335

[26] J.C. Dassun, A. Reyes, H. Yokoyama, P.B.E. Jireh, M. Dolendo, “Ordering points to identify the clustering structure algorithm in fingerprint-based age classification" Virtutis Incunabula, 2(1) 17-27, 2015.
[27] D. Comaniciu, P. Meer, "Mean shift: A robust approach toward feature space analysis" IEEE Transactions on Pattern Analysis and Machine Intelligence, 24(5) 603-619, 2002. DOI: 10.1109/34.1000236

[28] U.V. Luxburg, "A tutorial on spectral clustering" Statistics and Computing, 17(4) 395-416, 2007.

[29] D.A. Reynolds, "Gaussian mixture models" Encyclopedia of biometrics, 741, 2009.

[30] B.J. Frey, D. Dueck, "Clustering by passing messages between data points" Science, 315(5814) 972-976, 2007. DOI: 10.1126/science.1136800

[31] David Sculley, "Web-scale k-means clustering" in Proceedings of the 19th international conference on World Wide Web, pages 1177-1178, 2010. https://doi.org/10.1145/1772690.1772862

[32] E.B. Fowlkes, C.L. Mallows, "A method for comparing two hierarchical clusterings" Journal of the American Statistical Association, 78(383) 553-569, 1983.

[33] Y. Liu, Z. Li, H. Xiong, X. Gao, J. Wu, S. Wu, "Understanding and enhancement of internal clustering validation measures" IEEE Transactions on Cybernetics, 43(3) 982-994, 2013. DOI: 10.1109/TSMCB.2012.2220543

[34] S. Tsumoto, "Contingency matrix theory: Statistical dependence in a contingency table" Information Sciences, 179(11) 1615-1627, 2009. https://doi.org/10.1016/j.ins.2008.11.023

[35] A. Saxena, M. Prasad, A. Gupta, N. Bharill, O.P. Patel, A. Tiwari, M.J. Er, W. Ding, C. Lin, "A review of clustering techniques and developments" Neurocomputing, 267 664-681, 2017. https://doi.org/10.1016/j.neucom.2017.06.053

[36] A.K. Jain, M.N. Murty, P.J. Flynn, "Data clustering: a review" ACM computing surveys (CSUR), 31(3) 264-323, 1999. https://doi.org/10.1145/331499.331504

[37] P. Berkhin, "A survey of clustering data mining techniques" in Grouping multidimensional data, pp 25-71. Springer, 2006.

[38] A.Y. Ng, M.I. Jordan, Y. Weiss, "On spectral clustering: Analysis and an algorithm" in Advances in neural information processing systems, pp 849-856, 2002.

[39] G.C. Linderman, S. Steinerberger, "Clustering with t-sne, provably" SIAM Journal on Mathematics of Data Science, 1(2) 313-332, 2019. https://doi.org/10.1137/18M1216134

[40] D. Shepard, "A two-dimensional interpolation function for irregularly-spaced data" in Proceedings of the 1968 23rd ACM national conference, pp 517-524, 1968. https://doi.org/10.1145/800186.810616

[41] J. Venna, S. Kaski, "Neighborhood preservation in nonlinear projection methods: An experimental study" in International Conference on Artificial Neural Networks, pp 485-491, Springer, 2001.

[42] T. Kollig, A. Keller, "Efficient multidimensional sampling" Computer Graphics Forum, 21(3), 2002.

[43] D, Dua, C. Graff, UCI machine learning repository, 2019.

[44] B. Schölkopf, A. Smola, K. Müller, "Kernel principal component analysis" in International conference on artificial neural networks, pages 583-588, Springer, 1997. 